\title{
Computational models of basal-ganglia pathway functions: focus on functional neuroanatomy
}

\author{
Henning Schroll ${ }^{1,2,3,4 *}$ and Fred H. Hamker ${ }^{1,4 *}$ \\ Bernstein Center for Computational Neuroscience, Charitè - Universitätsmedizin Berlin, Berlin, Germany \\ 2 Department of Psychology, Humboldt-Universität zu Berlin, Berlin, Germany \\ ${ }^{3}$ Department of Neurology, Charitè - Universitätsmedizin Berlin, Berlin, Germany \\ ${ }^{4}$ Department of Computer Science, Chemnitz University of Technology, Chemnitz, Germany
}

\section{Edited by:}

Ahmed A. Moustafa, University of

Western Sydney, Australia

Reviewed by:

Ahmed A. Moustafa, University of

Western Sydney, Australia

Andrea Stocco, University of

Washington, USA

*Correspondence:

Fred H. Hamker and Henning

Schroll, Department of Computer

Science, Chemnitz University of

Technology, Straße der Nationen 62,

09111 Chemnitz, Germany

e-mail: fred.hamker@

informatik.tu-chemnitz.de;

henning.schroll@

informatik.tu-chemnitz.de
Over the past 15 years, computational models have had a considerable impact on basal-ganglia research. Most of these models implement multiple distinct basal-ganglia pathways and assume them to fulfill different functions. As there is now a multitude of different models, it has become complex to keep track of their various, sometimes just marginally different assumptions on pathway functions. Moreover, it has become a challenge to oversee to what extent individual assumptions are corroborated or challenged by empirical data. Focusing on computational, but also considering non-computational models, we review influential concepts of pathway functions and show to what extent they are compatible with or contradict each other. Moreover, we outline how empirical evidence favors or challenges specific model assumptions and propose experiments that allow testing assumptions against each other.

Keywords: dopamine, reinforcement learning, response selection, response timing, working memory, gating, stimulus-response association

\section{INTRODUCTION}

\subsection{INTRODUCTION TO THE CONCEPT OF BASAL-GANGLIA PATHWAYS}

Basal ganglia (BG) contain a variety of both glutamatergic and GABAergic fiber tracts. Why is BG organization that complex? Two influential theories, published more than 20 years back (Albin et al., 1989; DeLong, 1990), came up with a first idea: they proposed that BG control excitation and inhibition of cortex, therefore requiring two distinct pathways: a direct pathway (cortex $\rightarrow$ striatum $\rightarrow$ globus pallidus internus) was assumed to facilitate motor cortical activity, while an indirect pathway (cortex $\rightarrow$ striatum $\rightarrow$ globus pallidus externus $\rightarrow$ subthalamic nucleus $\rightarrow$ globus pallidus internus) was assumed to inhibit motor-cortical firing. These concepts provided an explanation of prominent BG motor disorders: over-activity of the excitatory direct pathway was assumed to result in overshoot of motor activity (as in Huntington's disease), while over-activity of the inhibitory indirect pathway was proposed to result in pathological motor inhibition (as in Parkinson's disease; Albin et al., 1989; DeLong, 1990). Inspired by this intuitive concept and the fact that it was later discovered to fail at explaining some prominent empirical findings (e.g., Marsden and Obeso, 1994), revised and extended models have been developed since. As part of this process, an additional, shorter route of the indirect pathway (cortex $\rightarrow$ striatum $\rightarrow$ globus pallidus externus $\rightarrow$ globus pallidus internus) has been proposed (Smith et al., 1998) as well as an additional hyperdirect pathway (cortex $\rightarrow$ subthalamic nucleus $\rightarrow$ globus pallidus internus; Nambu et al., 2002). If all of these pathways can be identified to fulfill distinct functions, the complexity of BG anatomy might be understood as a necessity to guarantee BG functionality.

According to general understanding, direct, indirect and hyperdirect BG pathways transmit cortical input to globus pallidus internus (GPi) and substantia nigra reticulata $(\mathrm{SNr})$, two largely analog BG output nuclei that tonically inhibit the thalamus (Figure 1). The direct pathway proceeds from cortex via striatum to GPi; information traversing this pathway has to pass a glutamatergic synapse first and a GABAergic synapse afterwards (Figure 1). Cortical input to the direct pathway thus reduces GPi firing which in turn increases activities in thalamus and cortex. The short indirect pathway passes from cortex to GPi via striatum and globus pallidus externus (GPe); synapses are glutamatergic, GABAergic and GABAergic, respectively. The long indirect pathway, in contrast, additionally passes through the subthalamic nucleus (STN) and contains an additional glutamatergic synapse (Figure 1). Cortical input to either of the two indirect pathways thus increases GPi firing. The hyperdirect pathway, finally, passes from cortex via STN to GPi and contains glutamatergic synapses only; cortical input to this pathway therefore increases GPi activity as well. Pathways are usually assumed to transmit information in a feed-forward manner; existing feedback-projections (e.g., from GPe to striatum or from STN to GPe; cf. Figure 1) are either assumed to not be part of these pathways or are assumed to be required for stabilization of information transmission only.

In the last decade, the rise of computational simulation techniques has boosted model development. Today, there is a multitude of different models, none of which yet accounts for all relevant empirical findings (section 9). Most of these models assume 


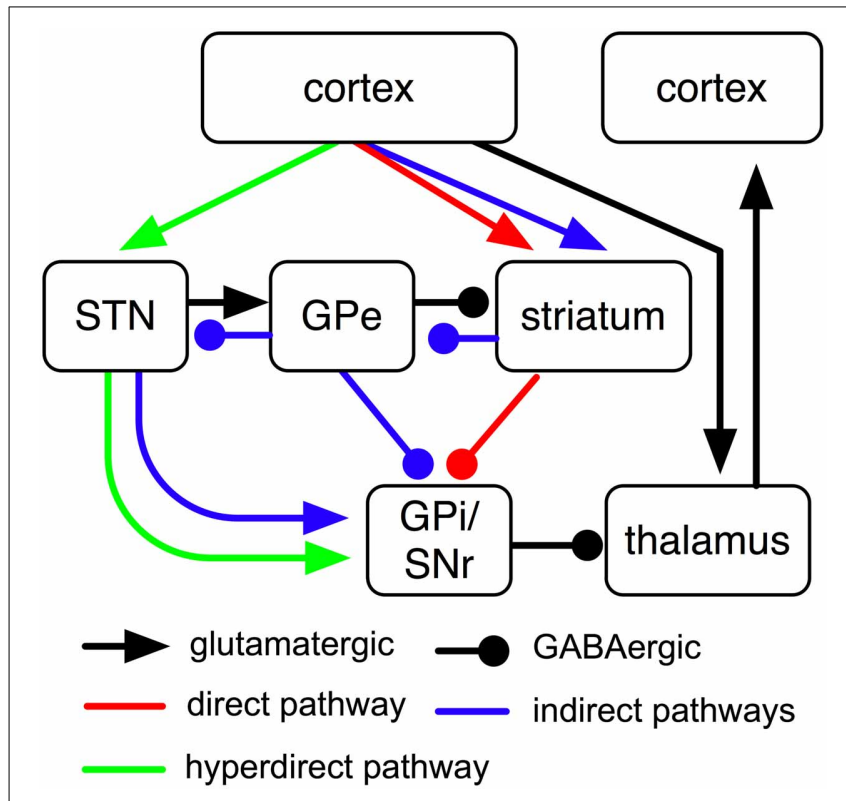

FIGURE 1 | Sketch of cortico-BG-thalamic fiber tracts and their subdivision into direct, indirect and hyperdirect BG pathways (cf. Bolam et al., 2000). Of the "indirect pathway," two routes have been proposed (Smith et al., 1998), the short one of which passes from GPe directly to GPi, while the longer one additionally passes through STN.

a clear anatomical separation between the different pathways. Although this is likely a simplification (Lévesque and Parent, 2005), physiological data corroborates the assumption of functionally separate pathways: electrical stimulation of cortex results in three temporally distinct changes of activity in GPi that can be traced back to the effects of direct, indirect and hyperdirect pathways, respectively (Nambu et al., 2000; Kita et al., 2006; Kita and Kita, 2011). Even if pathways are not built out of distinct sets of neurons, thus, they appear to be functionally separated.

\subsection{WHY COMPUTATIONAL MODELING?}

Most of the models and hypotheses we will review offer not just verbal and graphical descriptions, but an additional mathematical (i.e., computational) implementation. Such mathematical implementations offer important advantages, including, but not limited to the following: they allow computing the effects of non-linear interactions between simulated neurons that would be impossible to compute mentally. Moreover, they are innately precise, thus preventing fuzzy assumptions; if some of a model's various assumptions contradict each other or do not interact well, the model will fail to produce meaningful output. Finally, computational models produce predictions that do not immediately originate from their assumptions. Such predictions might, for instance, relate to model performance during specific behavioral tasks. As a note of caution, however, computational models are often hard to grasp intuitively: a set of mathematical formulas does not innately reveal what function a model serves. Rather, extensive and often iterative simulations are required to reveal these functions. To report and review computational models, thus, verbal and graphical descriptions of model assumptions and outputs are required as well. These, however, may suffer from lack of precision and in any case simplify a model's "real" computational details.

In the context of BG functioning, computational modeling has been particularly fruitful in recent years. The complexity of BG anatomy and physiology, in light of their substantial interactions with cortex, thalamus and other sub-cortical nuclei makes them a good target for computational modeling.

\section{ANATOMICAL AND PHYSIOLOGICAL CONSTRAINTS FOR INTERPRETATIONS OF PATHWAY FUNCTIONS 2.1. PATHWAY AFFERENTS FROM CORTEX AND THALAMUS}

The striatum (which is part of direct and indirect pathways) receives topographically organized inputs both from intratelencephalically-projecting cortical cells and from axon collaterals of cortical pyramidal-tract neurons (Figure 2A; Donoghue and Kitai, 1981; Lei et al., 2004; Parent and Parent, 2006; Shepherd, 2013). Cortico-striatal cells are predominantly located in cortical layer V, but also in layers II, III, and IV (Rosell and Giménez-Amaya, 1999). Striatal medium spiny neurons (MSNs) of the direct pathway have been shown to receive the majority of their inputs from intratelencephalically projecting cortico-striatal neurons, while striatal MSNs of the indirect pathways receive a greater proportion of inputs from axon collaterals of cortical pyramidal-tract neurons (Lei et al., 2004). The indirect pathways' inputs might thus largely consist of efference copies of motor output, informing this pathway about currently initiated responses.

Next to its MSNs, striatum contains cholinergic and several types of GABAergic interneurons (Tepper, 2010). While GABAergic interneurons receive extensive cortical input (Lapper et al., 1992; Kawaguchi, 1993; Ramanathan et al., 2002), cholinergic interneurons might receive more extensive input from thalamus than from cortex (Lapper and Bolam, 1992; Kawaguchi, 1993). Thalamic efferents to striatum are extensive and topographically organized (Berendse and Groenewegen, 1990; Lanciego et al., 2004).

STN (which gives rise to the hyperdirect pathway) receives topographically organized inputs from frontal and motor cortices (Hartmann-von Monakow et al., 1978; Afsharpour, 1985), again mainly from layer V (Canteras et al., 1990). Its cortical afferents have been described as deriving mainly from axon collaterals of cortico-fugal pyramidal-tract neurons (Giuffrida et al., 1985; Kita and Kita, 2012), thus potentially providing STN with efference copies of motor output. Potential inputs from sensory cortical areas have been both reported (Canteras et al., 1988) and repudiated (Afsharpour, 1985; Kolomiets et al., 2001). In any case, sensory cortices may modulate STN activity multi-synaptically via striatum and GPe of the long indirect pathway (Kolomiets et al., 2001). Like the striatum, STN receives topographically organized inputs from thalamus (Lanciego et al., 2004).

\subsection{PATHWAY CONDUCTION VELOCITIES}

Electrical stimulation of motor cortex results in triphasic changes of activity in GPi (Figure 2B; Nambu et al., 2000). Approximately $8 \mathrm{~ms}$ after stimulation of primary motor cortex, a fast excitation of GPi is observed that is followed by a short inhibition at about 

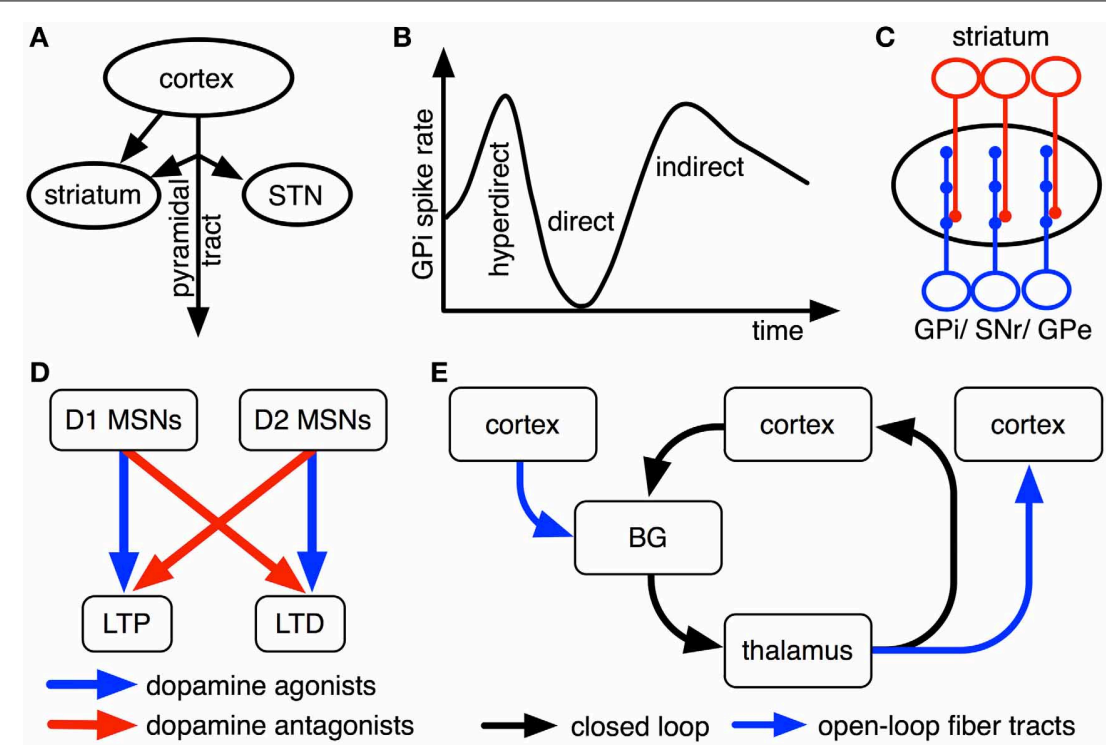

FIGURE 2 | Empirical findings that constrain interpretations on pathway functions. For illustrative purposes, all findings are shown as cartoons. (A) Striatum receives inputs from both intratelencephalically projecting cortical neurons and cortical pyramidal-tract neurons, while STN predominantly receives pyramidal-tract afferents (Donoghue and Kitai, 1981; Giuffrida et al., 1985; Lei et al., 2004; Parent and Parent, 2006; Kita and Kita, 2012). (B) Upon electrical stimulation of cortex, direct, indirect and hyperdirect pathways influence GPi activity with different latencies because of their different conduction velocities (Nambu et al., 2000; Kita and Kita, 2012). (C) Striatal cells innervate relatively small, circumscribed areas of GPi, SNr and GPe dendrites, whereas STN cells innervate these nuclei relatively broadly (Hazrati and Parent, 1992a,b). (D) Dopamine agonists and antagonists oppositely modulate long-term plasticity in cortico-striatal synapses of direct and indirect pathways (cf. Shen et al., 2008). (E) BG are organized in open and closed loops with cortex and thalamus (cf. Alexander et al., 1986; Joel and Weiner, 1994; Haber, 2003).
$21 \mathrm{~ms}$ after stimulation and a late excitation at about $30 \mathrm{~ms}$ after stimulation on average (Nambu et al., 2000). Chemical blocking of BG nuclei as well as parallel recordings in STN and GPe have shown that the fast excitation is caused by the hyperdirect pathway, the short inhibition by the direct pathway and the late excitation by the long indirect pathway (Nambu et al., 2000; Kita et al., 2006; Kita and Kita, 2011). The hyperdirect pathway's exceptionally fast response has been linked to unique properties of STN neurons, involving a slow decay of excitatory postsynaptic potentials (EPSPs) and a dynamic decrease in spike threshold after EPSPs (Farries et al., 2010; Kita and Kita, 2011).

\subsection{ARBORIZATION PATTERNS OF PATHWAY OUTPUTS}

BG pathways arborize differently broadly in GPi, thus affecting different numbers of GPi neurons: striatal neurons arborize with a high degree of specificity in globus pallidus in monkeys (Hazrati and Parent, 1992b), while STN neurons more uniformly excite large numbers of pallidal cells (Hazrati and Parent, 1992a,b; Figure 2C). Despite their different patterns of arborization, however, striatal and subthalamic cells were found to converge onto the same pallidal neurons in internal and external segments of globus pallidus (Hazrati and Parent, 1992a,c). Based on this evidence, the direct pathway and the short indirect pathway are usually assumed to influence relatively focused pallidal representations, whereas the hyperdirect pathway and the long indirect pathway likely exert relatively global effects (cf. Mink, 1996; Nambu et al., 2002; Brown et al., 2004; Nambu, 2004; Frank, 2006).

\subsection{DOPAMINERGIC IMPACTS ON BG PATHWAYS}

Synaptic plasticity in BG pathways is modulated by dopamine (Shen et al., 2008): while dopamine facilitates long-term potentiation (LTP) in cortico-striatal synapses of the direct pathway via D1-type dopamine receptors, it facilitates long-term depression (LTD) in cortico-striatal synapses of the indirect pathways via D2type dopamine receptors (Figure 2D; Shen et al., 2008). Phasic BG dopamine signals, as emitted by neurons of substantia nigra compacta $(\mathrm{SNc})$, have been hypothesized to encode error signals of reward prediction (Hollerman and Schultz, 1998): whenever an animal receives more reward than could be expected based upon previous reinforcement contingencies, dopamine neurons increase their firing above a low baseline rate; whenever less reward is received than could have been expected, firing decreases below this baseline. These findings inspired proposals that BG play an important role in reinforcement learning processes in the brain. Dopamine neurons, moreover, do not exclusively respond to rewarding events, but presumably also to salient non-rewarding and to aversive events (Bromberg-Martin et al., 2010). Dopaminergic effects on synaptic plasticity are well studied only for cortico-striatal synapses of direct and indirect pathways (Gerfen et al., 1990; Shen et al., 2008). For striatal outputs to GPe, GPi, and SNr, in contrast, the effects of dopamine have not yet been studied in similar detail. There are, however, hints that dopamine might modulate synaptic plasticity in these nuclei as well: all of them are innervated by axons of SNc dopamine neurons (Cossette et al., 1999; Gauthier et al., 1999); oral administration of the dopamine precursor levodopa 
modulates activity-dependent synaptic plasticity in $\mathrm{SNr}$ (Prescott et al., 2009). Moreover, it has been shown that SNr and entopeduncular nucleus (rat GPi equivalent) predominantly express D1 dopamine receptors (Boyson et al., 1986; Levey et al., 1993), that globus pallidus (rat GPe equivalent) expresses relatively high quantities of D2 receptors, but probably still more D1 dopamine receptors (Boyson et al., 1986; Levey et al., 1993) and that STN expresses both D1-type and D2-type receptors in considerable quantities (Flores et al., 1999). For connections from STN to SNr, moreover, D1 receptor agonists have been found to increase excitatory postsynaptic currents (EPSCs), whereas D2 receptor agonists decrease them (Ibañez-Sandoval et al., 2006). Based on these pieces of evidence, it has been assumed that increases in dopamine levels facilitate LTP along the entire direct pathway (involving both cortico-striatal and striato-GPi/ striato-SNr synapses), LTD along the entire short indirect pathway and LTP along the entire hyperdirect pathway (Schroll et al., 2013). Although this interpretation is consistent with existing empirical data, it has not yet been proven directly.

It is generally assumed that dopamine exerts additional shortterm effects on striatal activity that are in line with dopamine's effects on long-term plasticity: high dopamine levels are assumed to excite D1 MSNs of the direct pathway, while low dopamine levels are hypothesized to excite D2 MSNs of the indirect pathways (e.g., Wichmann and DeLong, 1996; Frank et al., 2004; Frank, 2005). Additionally, dopamine is assumed to modulate MSNs' sensitivity to glutamatergic synaptic inputs from cortex, again oppositely for D1 and D2 MSNs (Humphries et al., 2009). Empirically, dopamine has indeed been shown to modulate ion-channel conductances in the striatum (Calabresi et al., 1987; Lin et al., 1996). If, however, dopamine in fact spontaneously excites the direct and inhibits indirect pathways, remains to be shown (Calabresi et al., 2007). Similarly, it needs to be clarified to what extent spontaneous effects of dopamine fulfill a behaviorally relevant function on their own or might simply support dopaminergic effects on long-term plasticity. A detailed model of how dopamine affects the membrane properties of striatal MSNs has been provided by Humphries et al. (2009). Extending their model by known effects of dopamine on synaptic plasticity and including it, as a module, in systems-level models of cortico-BG-thalamic circuitry might help to understand the complex effects of dopamine on the functions of BG pathways.

\subsection{CORTICO-BG-THALAMIC LOOPS}

As outlined in Figure 1, BG are organized in loops with cortex and thalamus. It has been proposed that separate cortico-BGthalamic loops work in parallel and in relative independence of each other (Alexander et al., 1986). The number of independent loops is hard to estimate. Alexander et al. (1986) proposed the existence of at least five such loops (corresponding to motor, oculomotor, dorsolateral prefrontal, lateral orbitofrontal and anterior cingulate cortex). Frank et al. (2001) later suggested that each of these loops might be again subdivided into various sub-loops and estimated the human frontal cortex to contain around 20,000 such loops in total. Interestingly, the assumption of independent loops implies that each BG pathway has a variety of separate channels (i.e., one for each loop) and that each of these channels might subserve a different function (cf. Schroll et al., 2012). Thus, it might be more fruitful to search for superordinate principles of pathway functions than for specific pathway contributions related to individual loops. Along these lines, it has been distinguished between open and closed cortico-BG-thalamic loops (Figure 2E; Alexander et al., 1986; Joel and Weiner, 1994; Haber, 2003): while closed loops connect a particular area of cortex back to that same cortical area, open loops interconnect different areas of cortex. Anatomical crossovers between loops have indeed been described, in particular for cortico-striatal synapses (e.g., Inase et al., 1996; Takada et al., 1998; Calzavara et al., 2007) and cortico-thalamic synapses (Darian-Smith et al., 1999; McFarland and Haber, 2002). BG pathways might have entirely different functions in open loops than in closed loops. In particular, closed loops appear well fit for maintenance of information, while open loops might foster spread of information between cortical areas (Schroll et al., 2012; Trapp et al., 2012). For open loops, a hierarchy of information flow has been proposed that favors transmission of information from motivational via cognitive toward motor loops, but not vice versa (Haber, 2003).

\section{HYPOTHESES ON FUNCTIONAL CONTRIBUTIONS OF BASAL GANGLIA}

The above-mentioned pieces of evidence restrict the degrees of freedom for plausible hypotheses on pathway functions, but still leave a lot of interpretive freedom. Before reviewing hypothesized contributions of individual pathways, we will outline proposed functions of BG as an entirety.

\subsection{SELECTION MACHINE}

BG have been hypothesized to contribute to the selection of motor responses (e.g., Mink, 1996; Hikosaka et al., 2000; Gurney et al., 2001a,b; Frank et al., 2004; Nambu, 2004; Ashby et al., 2007; Schroll et al., 2012). Allowing for context-appropriate selection, they have moreover been assumed to establish and maintain associations between stimulus representations and response representations (Figure 3A; e.g., Reading et al., 1991; Packard and Knowlton, 2002). In line with these hypotheses, patients with BG disorders (i.e., Parkinson's disease and Huntington disease) are impaired in response selection (Lawrence et al., 1999; Wylie et al., 2009) and lesions of striatum result in impairments in acquiring stimulus-response rules (e.g., Reading et al., 1991; El Massioui et al., 2007). Recently, BG have been reported to be particularly involved in learning of stimulus- response associations, while they might be less important for execution of habitual stimulusresponse behavior (Antzoulatos and Miller, 2011; Waldschmidt and Ashby, 2011).

In a generalization of the selection hypothesis, BG have been proposed to select any cortical representation (rather than just motor programs), including internal cognitive and emotional states, based upon activation of any other representation (Trapp et al., 2012). In another generalization, BG have been assumed to establish associations not only between stimuli and responses, but between stimuli, responses and outcomes (Figure 3A; Redgrave and Gurney, 2006). 


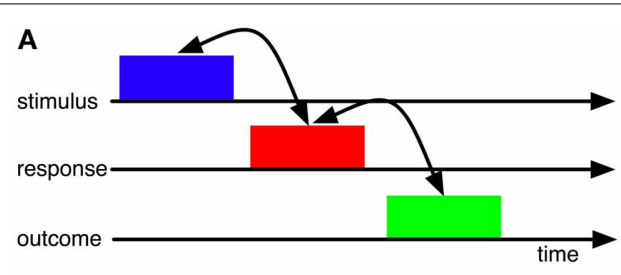

C

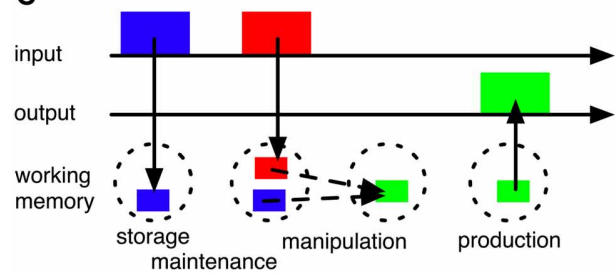

FIGURE 3 | Some influential concepts of BG functions. (A) BG may establish and maintain associations between stimuli and responses (or even between stimuli, responses and outcomes; Redgrave and Gurney, 2006) to allow context-based response selection. (B) BG may contribute to motor timing by providing initiation and termination signals for movements (Nambu, 2004) and by inhibiting premature responding (Frank, 2006). (C) BG may contribute to working memory functions, including gating of information into
B

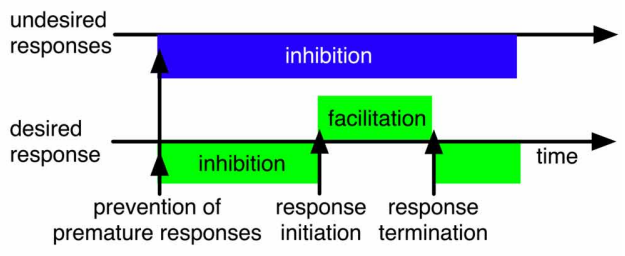

D

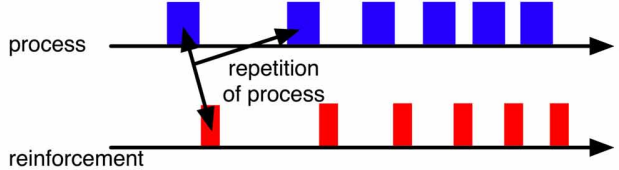

working memory (Gruber et al., 2006; O'Reilly and Frank, 2006), working-memory maintenance (Schroll et al., 2012) and production of information from working memory (Schroll et al., 2012). (D) BG may contribute to reinforcement learning processes, including, but not limited to reward-based learning, such that those processes or actions that result in reinforcements will be repeated (e.g., Houk et al., 1995; Berns and Sejnowski, 1998; Suri et al., 2001).

\subsection{PERformance OF SEQUENCES}

Based upon the idea that BG encode stimulus-response associations (section 3.1), they have been hypothesized to establish and execute sequences of motor processes by linking each single response of a sequence to its respective predecessor (Berns and Sejnowski, 1998; Nakahara et al., 2001). According to this hypothesis, BG interlink the different elements of a sequence in a stimulus-response manner, such that each performed "response" of a sequence serves as a "stimulus" for the following response. BG thus do not contain a single "overall" representation of each sequence, but an array of individual associations between its subsequent elements.

Evidence on BG involvement in sequence learning and execution has been provided for grooming in mammals (e.g., Berridge and Whishaw, 1992), song production in songbirds (e.g., Brainard and Doupe, 2000; Kao et al., 2005; Ölveczky et al., 2005) and sensorimotor production in humans (Doyon et al., 1997; Boecker et al., 1998).

\subsection{RESPONSE INITIATION AND TERMINATION}

BG have been hypothesized to provide initiation and termination signals for motor responding (Figure 3B; Nambu, 2004). According to this hypothesis, cortex sends a succession of corollary signals to the different BG pathways that ensure surroundinhibition of (premature) responses, response initiation and response termination, respectively. According to Nambu's (2004) hypothesis, BG determine the timing of already selected responses based on their corollary input signals from cortex (see also Mink, 1996); Nambu (2004), however, did not develop a computational model; we do not know of any such model that implements BG contributions to initiation and termination of motor responses in a loop linking the BG to primary motor cortex (M1).
Nambu's (2004) concept has been inspired by evidence of BG pathways' different conduction velocities as reviewed in section 2.2: stimulation of cortex first results in excitation, then inhibition, and finally again excitation of GPi. These three phases are assumed to correspond to inhibition of (premature) responses, response initiation and response termination, respectively (Nambu, 2004). However, pathway conduction velocities, by themselves, are no convincing proof of Nambu's (2004) assumptions: differences in conduction velocities are minute in magnitude and also relatively inflexible; they do not explain how response timing can be adapted to different contexts. Moreover, pathways not only have different conduction velocities but also receive different inputs that will likely set in at different times (section 2.1). These different set-ins of inputs may be far more decisive for latencies of pathway outputs than the pathways' conduction velocities. In line with this reasoning, Nambu (2004) hypothesized that pathways require temporally distinct corollary inputs from cortex to properly initiate and terminate responses.

\subsection{WORKING MEMORY GATING AND MAINTENANCE}

In the cognitive domain, BG have been hypothesized to control working-memory processes (Figure 3C). According to one proposal (O'Reilly and Frank, 2006), they guard the gate to working memory and thereby determine which stimuli will be maintained. Learning which stimuli to gate through initially requires random gating of working-memory contents according to this proposal. According to a different proposal (Schroll et al., 2012), BG are part of a working-memory maintenance system, allowing for reverberation of information in cortico-BG-thalamic loops. Here, BG are assumed to both determine which pieces of information enter working memory and to contribute to their actual maintenance. This model does not require an initially random selection of working-memory contents, but relies on shaping to 
learn complex working-memory paradigms. There is ample evidence on BG involvement in working memory tasks, both from human (Lewis et al., 2004; Alberts et al., 2008; Hershey et al., 2008; Moustafa et al., 2008b; Landau et al., 2009) and animal subjects (Levy et al., 1997). Empirical differentiation between the two hypotheses, however, is not yet possible as will be outlined in section 4.5 .

\subsection{DIMENSIONALITY REDUCTION}

BG have been proposed to establish a focus on relevant (salient) information by reducing dimensionality of cortical information (Bar-Gad et al., 2000). Based on dopaminergic reinforcement signals, BG are assumed to learn efficient compression of information in such a way that its approximate reconstruction remains possible. In line with empirical data (Nelson et al., 1992; Nini et al., 1995; Bar-Gad et al., 2000), the model by Bar-Gad et al. (2000) predicts that correlations between neuronal activities decrease from cortex to globus pallidus. It might moreover explain why cortex contains far more neurons than striatum, which again contains far more neurons than GPi/SNr (Oorschot, 1996).

\subsection{REINFORCEMENT LEARNING}

Most computational models assume that BG pathways contribute to reinforcement learning (e.g., Berns and Sejnowski, 1998; Brown et al., 2004; Frank, 2006; O'Reilly and Frank, 2006; Ashby et al., 2007; Stocco et al., 2010; Schroll et al., 2012). According to this hypothesis, BG adapt behavior in such a way that reinforcements are maximized (Figure 3D). Specifically, they are assumed to foster repetition of those actions, emotions and cognitive processes that result in reinforcements.

Under the umbrella term "reinforcement learning," BG have been proposed to learn from unexpected rewards (e.g., Suri et al., 2001; Brown et al., 2004; Ashby et al., 2007; Vitay and Hamker, 2010), from punishments (e.g., Frank et al., 2004), and, generally, from unexpected sensory events (Redgrave and Gurney, 2006). The latter generalization implies that BG may learn any novel association between stimuli, actions and outcomes, even if not followed by reward. Via such a mechanism, animals and humans might learn contingencies that are relevant for obtaining positive outcomes in the future: for instance, they might find out that a particular action results in access to a safe sleeping place, even when currently foraging for food (cf. Redgrave and Gurney, 2006).

The reinforcement-learning hypothesis is based on findings that phasic dopamine signals in BG encode error signals of reward prediction (Hollerman and Schultz, 1998) and other salient unexpected events (e.g., Horvitz et al., 1997; Rebec, 1998), where value and salience of events might be signaled by distinct dopamine systems (Bromberg-Martin et al., 2010). The hypothesis is further based on evidence that dopamine modulates synaptic plasticity in BG (Shen et al., 2008). However, synaptic plasticity is well investigated only for cortico-striatal fibers. Some computational models thus limit dopamine-modulated learning processes to these cortico-striatal fibers (e.g., Brown et al., 2004; Ashby et al., 2007; Guthrie et al., 2009; Moustafa and Gluck, 2011), while others, more daringly, assume them to occur along more extensive parts of BG pathways (e.g., Vitay and Hamker, 2010; Schroll et al., 2012). In a particularly strong version of the reinforcementlearning hypothesis, BG refrain from processing as a particular function becomes automatized, i.e., after this function has been reliably learned via reinforcements (Ashby et al., 2007). This hypothesis is corroborated by single cell data from monkeys (Antzoulatos and Miller, 2011) and functional imaging data from humans (Waldschmidt and Ashby, 2011). Automatic functioning has instead been assumed to rely on cortico-cortical or cortico-thalamo-cortical connections (Ashby et al., 2007; Schroll et al., 2013). These connections might allow for faster information transfer because of fewer synaptic contacts than the route through the BG and might thus explain reduced reaction times in automatized tasks (Ashby et al., 2007).

The reinforcement-learning hypothesis is a meta-perspective that is fully compatible with any of the hypotheses outlined in sections 3.1 to 3.5 , since it refers to how BG pathways arrive at a particular function and not to what that function is. In fact, all of the hypotheses outlined in sections 3.1 to 3.5 may be correct since BG might flexibly learn to establish exactly those functions that result in reinforcements in a given learning context. Empirical evidence on BG involvement in reinforcement learning is extensive for both animals (e.g., Featherstone and McDonald, 2004; El Massioui et al., 2007; Antzoulatos and Miller, 2011) and humans (e.g., Frank et al., 2004; Tanaka et al., 2004; Schönberg et al., 2007; Moustafa et al., 2008a). Computational models are particularly suitable for formalizing (and then simulating) reinforcementlearning processes because of these processes' iterative nature.

\section{PROPOSED FUNCTIONS OF THE DIRECT PATHWAY}

As outlined in sections 2.3 and 2.4, the direct pathway facilitates cortical activity, it is strengthened by dopamine and its arborization is focused rather than divergent. In the following sub-sections 4.1 to 4.6 , we will review the direct pathway's proposed functions in detail. Sections 5 and 6 will then cover proposed functions of indirect and hyperdirect pathways. To provide a quick overview, Table 1 summarizes which models interpret which aspects of BG anatomy.

\subsection{GLOBAL MOTOR FACILITATION}

Early non-computational models (Albin et al., 1989; DeLong, 1990) as well as a recent computational model of BG pathways (Stocco et al., 2010) proposed that the direct pathway unspecifically facilitates motor activity. And indeed, it has been confirmed optogenetically that stimulation of striatal MSNs of the direct pathway results in increased locomotion in mice (Kravitz et al., 2010). If this is a (relatively) unspecific effect, however, remains to be shown. The relatively sparse arborization of striatal MSNs in GPi intuitively challenges the global-facilitation hypothesis, although the degree of sparseness cannot yet be interpreted in functional terms and thus is no proof against the hypothesis.

\subsection{SPECIFIC MOTOR FACILITATION}

Mink (1996) proposed that the direct pathway specifically facilitates desired responses (rather than motor activity per se). This hypothesis therefore directly contradicts the global-facilitation 


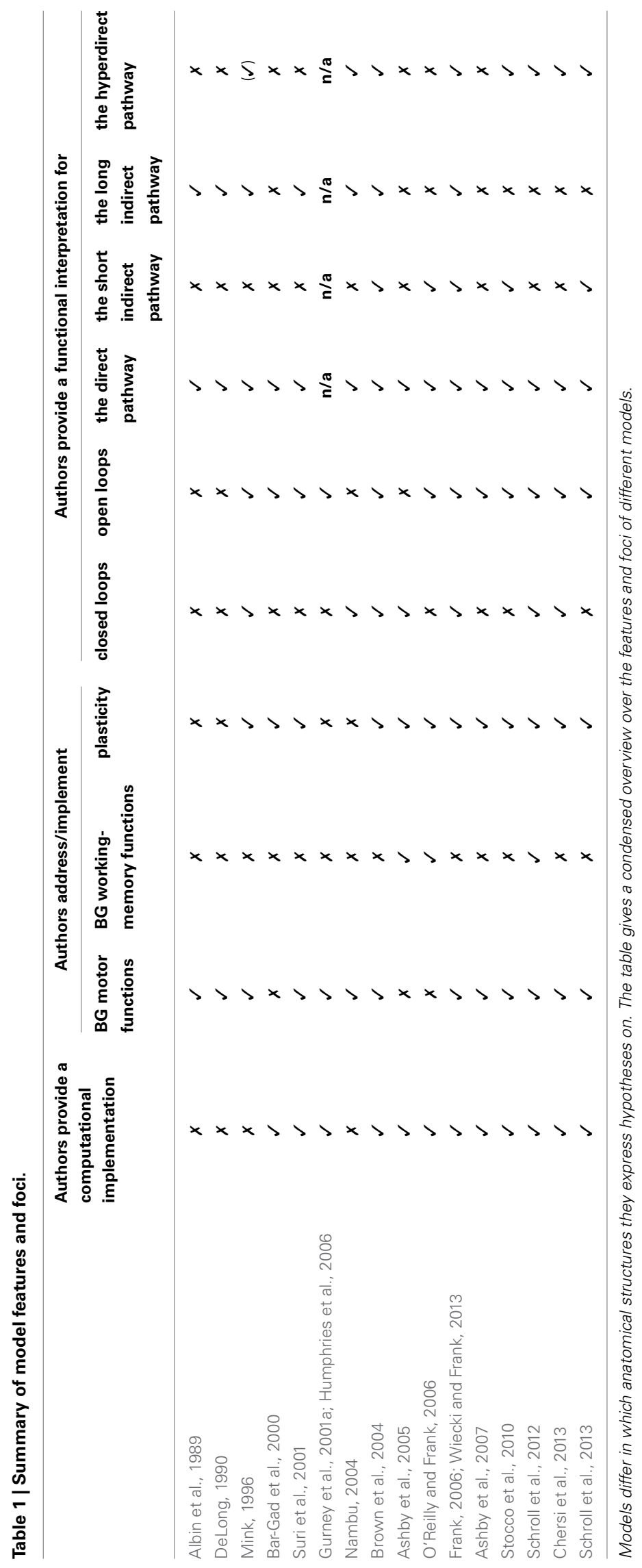


hypothesis outlined in section 4.1. Recent computational models mostly follow Mink's (1996) suggestion (e.g., Gurney et al., 2001a,b; Suri et al., 2001; Brown et al., 2004; Frank, 2005; Ashby et al., 2007; Schroll et al., 2012) and applied it to cognitive operations as well (e.g., O'Reilly and Frank, 2006; Schroll et al., 2012). Because of the multitude of parallel cortico-BG-thalamic loops (section 2.5), it indeed appears likely that different channels of the direct pathway may simultaneously facilitate different types of representations (Schroll et al., 2012). Most computational models moreover hypothesize that the direct pathway learns to facilitate specific cortical representations based on rewards (e.g., Suri et al., 2001; Brown et al., 2004; Frank, 2005; Ashby et al., 2007; Schroll et al., 2012). We do not know of any empirical data that favors the specific-facilitation hypothesis over the global-facilitation hypothesis or vice versa.

\subsection{STIMULUS-RESPONSE MAPPING}

The direct pathway has been hypothesized to facilitate specific motor programs only if they are appropriate in a given stimulus context (e.g., Brown et al., 2004; Ashby et al., 2007; Vitay and Hamker, 2010; Schroll et al., 2012). This hypothesis is a more specific version of the specific-facilitation hypothesis outlined in section 4.2. It says that the direct pathway connects specific stimulus representations to specific response representations (i.e., motor programs) and then facilitates a particular motor program only if the corresponding stimulus representation is active. The stimulus-response hypothesis relies on the existence of open cortico-BG-thalamic loops that interlink cortical areas involved in stimulus processing with areas involved in motor responding. And indeed, striatal areas that receive inputs from both primary somatosensory and primary motor cortices have been reported (Flaherty and Graybiel, 1993). Clear evidence of open corticoBG-thalamic loops that connect visual or auditory cortices to primary motor cortex, however, has not yet been shown, although both higher-order visual and higher-order auditory cortices are known to project to striatum (LeDoux et al., 1991; Bordi and LeDoux, 1992; Baizer et al., 1993).

In a generalization of the stimulus-response hypothesis, internal states (like emotions, mental images or abstract cognitive concepts) may serve as stimuli for stimulus-response associations as well. In an even broader generalization, the direct pathway may interlink any two cortical representations (cf. Trapp et al., 2012), potentially in a hierarchy from emotional via motivational, cognitive and premotor to motor regions (Haber, 2003).

Ashby and Crossley (2011) proposed that striatal cholinergic interneurons take part in the establishment of stimulus-response associations. They suggest that these interneurons tonically inhibit striatal MSNs of the direct pathway in the absence of stimulus inputs and that a stimulus-contingent release of inhibition is required for a direct-pathway induced activation of responses.

\subsection{TEMPORALLY PRECISE INITIATION OF RESPONSES}

Nambu (2004) hypothesized that the direct pathway determines the point in time when a particular response is initiated. According to his concept, the cortex selects an appropriate response and then first sends a corollary signal to the hyperdirect pathway, which globally inhibits all motor programs. Briefly afterwards, a second corollary signal to the direct pathway initiates a specific motor response at the appropriate point in time. This response-initiation hypothesis is, in its core, compatible with the specific-facilitation hypothesis (section 4.2) and the stimulus-response hypothesis (section 4.3): the direct pathway may well select appropriate responses (potentially based upon stimulus input) and also determine the exact time at which they are initiated. The specifics of these hypotheses, however, are incompatible: according to Nambu (2004), the cortex decides for a response, while it is the direct pathway and its specific connectivity according to the other two proposals.

Empirical evidence for the response-initiation hypothesis comes from patients with Parkinson's disease, a BG disorder that goes along with decreased activation of the direct pathway (but also with increased activation of the indirect pathways; Kravitz et al., 2010; Kita and Kita, 2011): as predicted by the responseinitiation hypothesis, Parkinson's disease patients are impaired in initiating movements (Bloxham et al., 1984; Carli et al., 1985; Hikosaka et al., 1993), but not in completing them (Carli et al., 1985) or in performing pre-programmed movements (Bloxham et al., 1984).

The response-initiation hypothesis as well as, in fact, any of the hypotheses outlined in sections 4.1 to 4.3 , has been challenged based on reports that BG activation mostly occurs only after overt responses are visible (e.g., Aldridge et al., 1980; Jaeger et al., 1995). However, some BG neurons do become active before EMG onset (Jaeger et al., 1995) and reports of delayed BG activity may result from the specific study designs involved: motor responses were trained for extensive amounts of time in these studies, before BG were recorded. Recent evidence, however, points at an important role of the BG in initiating responses only while new response patterns are being learned (Antzoulatos and Miller, 2011; Waldschmidt and Ashby, 2011).

\subsection{WORKING-MEMORY GATING AND MAINTENANCE}

The direct pathway has been suggested to play an important role in working-memory functions. In particular, it has been suggested to gate information into working memory (Gruber et al., 2006; O'Reilly and Frank, 2006), but also to contribute to working-memory maintenance itself (Ashby et al., 2005; Schroll et al., 2012). According to the former hypothesis, the direct pathway is required for gating information into PFC, where it is then maintained independent of direct-pathway activity. According to the latter hypothesis, in contrast, maintenance of workingmemory content requires reverberation of activity in cortico-BGthalamic loops, explicitly involving the direct pathway. Empirical evidence does not clearly favor one interpretation over the other. The former hypothesis predicts a phasic change in direct-pathway activity only while working-memory content is gated, while the latter predicts sustained activity over delay periods of workingmemory tasks. In favor of the latter hypothesis, a subset of striatal neurons has been empirically shown to exhibit sustained activities over delay periods of a spatial delayed response task (Cromwell and Schultz, 2003) and of a delayed saccade task (Hikosaka et al., 1989). In favor of the former hypothesis, however, the caudate nucleus of the striatum has been found more active during working memory manipulation than during working memory 
maintenance in a functional magnetic resonance imaging (fMRI) study (Lewis et al., 2004).

\subsection{DIMENSIONALITY REDUCTION}

The direct pathway has been proposed to perform the dimensionality reduction process outlined in section 3.5. No other BG pathway is assumed to take part in this process.

\section{PROPOSED FUNCTIONS OF LONG AND SHORT INDIRECT PATHWAYS}

Two indirect pathways have been described, both of which inhibit cortical activity (Figure 1): a short route passes from GPe directly to GPi and arborizes there rather sparsely, while a longer route passes from GPe to STN and further from STN to GPi where it arborizes rather profusely (section 2.3). High dopamine levels foster LTD in cortico-striatal synapses that belong to these indirect pathways, while low dopamine levels facilitate LTP (section 2.4). As most models implement only one of the two indirect pathways, their interpretations might not be specific to the particular pathway included. To highlight the often-neglected fact that these pathways might establish entirely different functions, however, we nevertheless explicitly differentiate between the two pathways.

\subsection{GLOBAL INHIBITION OF MOTOR PROGRAMS (LONG INDIRECT PATHWAY)}

In early non-computational models, the long indirect pathway is assumed to globally inhibit motor behavior (Albin et al., 1989; DeLong, 1990). This hypothesis is in good agreement with the long indirect pathway's relatively global effects on GPi as outlined in section 2.3. Functional evidence for this hypothesis comes from an optogenetic study, where stimulation of striatal MSNs of the indirect pathways resulted in decreased motor initiation and increased bradykinesia (Kravitz et al., 2010). However, this study did not differentiate between long and short indirect pathways. Moreover, striatal MSNs of the indirect pathways were stimulated relatively globally which may be expected to cause global effects on behavior even if the long indirect pathway does not act as globally as hypothesized.

\subsection{INHIBITION OF SPECIFIC MOTOR PROGRAMS (SHORT INDIRECT PATHWAY)}

Based on its sparse arborization, the short indirect pathway has been suggested to inhibit specific motor programs (Brown et al., 2004; Frank et al., 2004; Frank, 2005; Schroll et al., 2013). More specifically, it has been hypothesized to learn this inhibition based on unfavorable outcomes, including omissions of expected rewards (e.g., Brown et al., 2004; Schroll et al., 2013) and aversive events (Frank et al., 2004). The chain of events between the occurrence of these unfavorable outcomes and the inhibition of motor programs is assumed to be the following: unfavorable outcomes cause phasic reductions in BG dopamine levels, which then activate the short indirect pathway to suppress the response that had resulted in the unfavorable outcome. Empirically, phasic decreases in dopamine have indeed been shown to strengthen cortico-striatal synapses of the indirect pathways (Shen et al., 2008). Moreover, it has indeed been shown that omissions of expected rewards result in phasic decreases in dopamine activity (Hollerman and Schultz, 1998); it thus appears plausible that the short indirect pathway inhibits responses based on omissions of expected rewards. The consequences of aversive events, however, might be more complex: while some SNc neurons indeed become less active following aversive events, others increase their activity (Matsumoto and Hikosaka, 2009). Insofar as $\mathrm{SNc}$ neurons respond differently to reward omissions and aversive events, it remains speculative if the short indirect pathway inhibits responses based on aversive events as well. In favor of such an effect, blocking of the indirect pathways (but not the direct pathway) in genetically modified mice has been shown to result in impaired shock avoidance (Hikida et al., 2010). Moreover, Frank et al. (2004) showed that Parkinson's disease patients (who suffer from dopamine loss) learn better from negative as opposed to positive outcomes than healthy controls (but see Shiner et al., 2012, for a challenge of their conclusions). Thus, the short indirect pathway may well learn to inhibit motor programs based both on omissions of rewards and on aversive events.

Omissions of expected rewards occur primarily during reversal learning and extinction (i.e., when expected stimulus-responsereward associations are no longer valid). In the model by Schroll et al. (2013), therefore, the short indirect pathway inhibits specific responses specifically during reversal learning. Pharmacological studies indeed show that D2 receptor agonists (which predominantly target indirect pathways; cf. section 2.4) impair reversal learning in humans (Mehta et al., 2001). Also, D2-type receptor antagonists (but not D1-type antagonists) result in reversallearning deficits in non-human primates (Lee et al., 2007). By assuming that both D2 agonists and D2 antagonists render D2 receptors insensitive to phasic changes in physiologically generated dopamine signals, these findings are in line with Schroll et al.'s (2013) hypothesis: according to their model, suppression of previously correct responses during reversal learning requires task-related, phasic unbinding of dopamine at D2 receptors after omissions of expected rewards. Both D2 agonists and D2 antagonists can be assumed to impair such a task-related unbinding. None of the above-cited studies, however, distinguished between long and short routes of the indirect pathway. It thus remains to be established to what extent it is indeed the short route that inhibits specific responses during reversal learning.

\subsection{TERMINATION OF EXECUTED RESPONSES (LONG INDIRECT PATHWAY)}

In consideration of the long indirect pathway's relatively slow conduction velocity (section 2.2), this pathway has been hypothesized to provide termination signals for motor execution (Nambu, 2004). In line with this hypothesis, GPi cells show a triphasic change of activity in response to cortical stimulation: an early excitation via the hyperdirect pathway is followed by a brief inhibition via the direct pathway and by a late excitation via the long indirect pathway (Figure 2B). According to the termination hypothesis, the late excitation terminates those motor responses that are initiated via the intermediate inhibition. Acknowledging the long indirect pathway's relatively broad effects 
on GPi (section 2.3), the proposed termination signal has been assumed to act relatively globally (Nambu, 2004).

The global-response-termination hypothesis is well compatible with the hypothesis that the long indirect pathway globally inhibits responses (section 5.1). Response termination, however, requires a delay in suppression such that responses can be initiated first.

We do not know of any direct functional evidence for a termination function of the long indirect pathway.

\subsection{DEFERRAL OF SELECTED PLANS (SHORT INDIRECT PATHWAY)}

According to Brown et al. (2004), the short indirect pathway defers execution of specific selected responses until appropriate. This hypothesis is an extension of the specific-inhibition hypothesis outlined in section 5.2 with regard to the dimension of time. Response deferral is assumed to be no built-in function of the short indirect pathway, but needs to be learned from omissions of expected rewards. In other words, the default is to not defer chosen plans. If, however, premature release of a response via the direct pathway results in reward omission, the short indirect pathway learns to inhibit this response (Brown et al., 2004). According to the model, response deferral is learned in a contextbased way by associating the deferral to any stimulus input that might be present during the deferral period. Thalamo-striatal feedback to the short indirect pathway is hypothesized to inform the short indirect pathway which response was selected before reward omission so that exactly this response can be inhibited. We do not know of any empirical evidence corroborating the deferral hypothesis.

\subsection{SURROUND-INHIBITION OF COMPETING MOTOR PROGRAMS}

Both indirect pathways have been hypothesized to establish a surround inhibition of unwanted motor programs during motor responding (Mink, 1996; Stocco et al., 2010). Mink (1996) proposed a particular role of the STN in this respect, thus referring to the long indirect pathway (but also to what is known today as the hyperdirect pathway). In contrast, Stocco et al. (2010) proposed that the short route of the indirect pathway is involved. Neither Mink (1996) nor Stocco et al. (2010) hypothesized on how broad the "space" of suppressed competing motor programs may be (i.e., if every other motor program would be inhibited or just a set of particularly strong competitors). Therefore, arborization patterns do not provide any consistent clue whether an involvement of the short or the long route is more realistic. As a challenge to both hypotheses, however, the effects of dopamine on longterm plasticity in cortico-striatal MSNs of the indirect pathways are exactly opposite those observed in cortico-striatal MSNs of the direct pathway (section 2.4; Shen et al., 2008). Thus, when facilitation of a response via the direct pathway is strengthened by dopamine, surround inhibition of its competitors may not be strengthened as well; center facilitation and surround inhibition can not be established at the same time unless the activities of dopamine neurons that target the "center" increase, while those that target the "surrounds" may simultaneously decrease. Since such an effect has not yet been reported, the hyperdirect pathway might be a more suitable candidate for surround inhibition than any of the indirect pathways (cf. section 6.4).

\subsection{CONTROL SYSTEM}

Challenging the subdivision of BG fiber tracts into direct, indirect and hyperdirect pathways, BG have been proposed to consist of a selection pathway, containing what is referred to as direct and hyperdirect pathways in this review, and of a control pathway, vaguely consisting of what is termed long and short indirect pathways here (Gurney et al., 2001a,b; Humphries et al., 2006). More specifically, the control pathway is assumed to consist of the full short indirect pathway, the long indirect pathway up to the STN and an additional route from cortex via STN to GPe (Gurney et al., 2001a). According to Gurney et al. (2001a,b), the control pathway does not have a separable function itself, but rather supports direct and hyperdirect pathways in selecting responses. Among its functions, the control pathway is hypothesized to regulate the amount of activity in STN (and thereby also in GPi): according to the model, motor selection requires that the amount of global motor inhibition is neither so strong that it overrules any facilitation of a specific motor program via striatum, nor so weak that multiple responses are selected simultaneously. By regulating the activity of STN cells and thereby the amount of global motor inhibition, the control pathway ensures an appropriate balance between excitation and inhibition such that neither too many nor too few responses are released simultaneously.

A similar concept is based on the architecture of direct and long indirect pathways as specified in this review (cf. Figure 1): in Suri et al.'s (2001) model, the long indirect pathway is hypothesized to globally increase motor inhibition such that only significant contributions of the direct pathway will result in cortical activation, while insignificant direct-pathway effects will be suppressed.

\subsection{CLOSING THE GATE TO WORKING MEMORY (SHORT INDIRECT PATHWAY)}

With regard to working memory, the short indirect pathway has been proposed to prevent gating of information into workingmemory storage systems (O'Reilly and Frank, 2006). According to this hypothesis, the short indirect pathway and the direct pathway oppose each other in a push-and-pull manner (section 7.1) to forbid or allow gating of information into working memory, respectively. The hypothesis is structurally similar to, and therefore compatible with, the idea that the short indirect pathway inhibits (gating of) specific motor programs (section 5.2); these functions could be performed by different cortico-BG-thalamic loops. Although there is evidence for BG involvement in working-memory functions (Levy et al., 1997; Lewis et al., 2004; Landau et al., 2009), there is, to the best of our knowledge, no data on the specific gating function of the short indirect pathway proposed by O'Reilly and Frank (2006).

\section{PROPOSED FUNCTIONS OF THE HYPERDIRECT PATHWAY}

As outlined in sections 2.2 and 2.3, the hyperdirect pathway excites GPi relatively fast and relatively globally. A major proportion of its inputs derives from axon collaterals of pyramidal tract neurons (section 2.1), while synaptic plasticity in this pathway is not yet well understood (section 2.4). 


\subsection{PREVENTION OF PREMATURE RESPONSES}

Based on its fast and relatively global effects on GPi, the hyperdirect pathway has been proposed to globally prevent premature responses until response selection has been completed (Frank, 2006; Stocco et al., 2010). Along these lines, the hyperdirect pathway has been predicted to be particularly vital in situations where extensive response conflict occurs (Frank, 2006; Frank et al., 2007), i.e., whenever multiple competing motor programs are simultaneously active in premotor cortex. Recordings of STN activity during high-conflict and low-conflict choices have been performed to investigate this prediction. A typical paradigm starts with a couple of training trials, in which subjects are presented with pairs of stimuli (e.g., A-B or C-D) and have to choose one stimulus of each pair. Each stimulus is associated with a fixed reward probability across trials (e.g., A: 20\%-B: $80 \%$, and C: 30\%-D: 70\%).

Being instructed to maximize rewards, subjects are supposed to learn to choose the stimulus of each pair that provides better average outcomes (i.e., B and D respectively, in our example). In subsequent test trials, pairs are shuffled such that high-conflict pairs (e.g., B-D) and low conflict pairs (e.g., A-D) may be presented. Using such a task, human patients $\mathrm{ON}$ deep brain stimulation (DBS) of the STN (which inhibits spiking activity in STN, Gradinaru et al., 2009, and thus eliminates task-related information processing in this nucleus) have been shown to make faster decisions under high response conflict than patients OFF DBS (Frank et al., 2007). Moreover, intracranial EEG recordings from DBS electrodes have revealed differences in STN oscillatory activity between high-conflict and low-conflict trials (Cavanagh et al., 2011), thus arguing for a contribution of STN to conflict processing, in line with Frank et al.'s (2007) prediction.

\subsection{STOPPING PREPARED RESPONSES BEFORE EXECUTION}

Along similar lines, the hyperdirect pathway has been hypothesized to globally inhibit prepared responses when a stop signal is shown before response execution (Aron and Poldrack, 2006; Aron, 2011; Wiecki and Frank, 2013). This hypothesis is fully compatible with the premature-response-inhibition hypothesis outlined in section 6.1; the hyperdirect pathway may flexibly switch between both of these functions as required by context. Both functions require fast and global inhibition of motor programs, which would fit well with the hyperdirect pathway's fast conduction velocity and its relatively global effects on GPi. Indeed, not just the premature-response-inhibition hypothesis, but also the stop hypothesis is corroborated by empirical evidence: in an fMRI study, STN (which is part of the hyperdirect pathway-but also of the long indirect pathway) has been shown to be more active in humans in stop trials than in go trials of a stop-signal task (Aron and Poldrack, 2006). In that same study, STN has been found more active in subjects that show a fast inhibition of responses after a stop cue (i.e., a fast stop-signal reaction time, SSRT) than in subjects with slow response inhibition. Contrarily, however, another fMRI study reported smaller STN activity in fast-inhibiting subjects than in slow inhibitors (Ray Li et al., 2008). As a challenge to the stop-signal hypothesis, PD patients (whose STN activity is increased; Kreiss et al., 1997;
Huang et al., 2007), show slower (instead of faster) inhibition of responses in stop-signal tasks (Gauggel et al., 2004).

\subsection{DEACTIVATION OF BG TO ALLOW FOR TOP-DOWN CONTROL}

The hyperdirect pathway has been hypothesized to subdue BG influences on motor cortex in order to allow for top-down PFC control over motor-cortical activities (Chersi et al., 2013). According to this hypothesis, PFC inputs to the hyperdirect pathway decrease activities of all GPi/ $\mathrm{SNr}$ neurons to similar levels via inhibitory interneurons, thereby overruling any response-activating effects caused by the direct pathway and preventing task-related BG outputs (Chersi et al., 2013). PFC may then control motor-cortical activities itself. By proposing that the hyperdirect pathway globally overrules any effects of the direct pathway, the deactivation hypothesis has a common assumption with the premature-response-inhibition hypothesis (section 6.1) and the response-stopping hypothesis (section 6.2). However, the deactivation hypothesis specifies that the hyperdirect pathway decreases activities in BG output nuclei (via inhibitory interneurons; Chersi et al., 2013), while the other two hypotheses assume it to increase firing in these nuclei. Since the major effect of the hyperdirect pathway on GPi/SNr is known to be excitatory (cf. Figure 1), we do not consider the deactivation hypothesis to be particularly plausible in this respect. It might, however, still hold true in its core: a global increase in GPi firing could deactivate BG to allow for top-down control just as well.

\subsection{SURROUND INHIBITION OF COMPETING MOTOR PROGRAMS}

Just like the indirect pathways (section 5.5), the hyperdirect pathway has been hypothesized to establish surround-inhibition of unwanted motor programs during responding (Gurney et al., 2001a; Nambu, 2004; Humphries et al., 2006; Schroll et al., 2013). Two versions of this hypothesis exist: according to the first, the hyperdirect pathway inhibits only those responses that compete for execution with the desired response, but not the desired response itself which is instead facilitated via the direct pathway (Schroll et al., 2013). According to the second version, the hyperdirect pathway globally inhibits all motor programs, including the desired one, which is distinguished only by its additional activation via the direct pathway (Gurney et al., 2001a; Nambu, 2004; Humphries et al., 2006).

Both hypotheses well reflect the different arborization patterns of direct and hyperdirect pathways, which have been found sparse and profuse, respectively (section 2.3). We don't know of any empirical study investigating the strict surroundinhibition hypothesis against the more unspecific surroundinhibition hypothesis.

\subsection{WORKING-MEMORY UPDATE}

The hyperdirect pathway has been hypothesized to clear information from working-memory and to thus allow for updating of working-memory content (Schroll et al., 2012). According to this hypothesis, the hyperdirect pathway breaks reverberation of activity in cortico-BG-thalamic loops (which is assumed to be the neuronal basis of working-memory maintenance, section 4.5). Empirical evidence for this hypothesis is yet rather 
unspecific: DBS of the STN in Parkinson's disease patients (which is assumed to inhibit spiking activity in this nucleus; Gradinaru et al., 2009) impaired working-memory performance in a spatial delayed response task (Hershey et al., 2008) and in an n-back task (Alberts et al., 2008); to what extent these effects were in fact produced by failures in updating working-memory content, however, or may have been caused by other types of errors, was not delineated. The working-memory-update hypothesis may be generalized to an involvement of the hyperdirect pathway in updating of any information that may be maintained in closed cortico-BG-thalamic loops.

\section{PROPOSED INTERACTION PATTERNS BETWEEN PATHWAYS}

Having reviewed prominent hypotheses on pathway functions, we will now outline in how far these hypotheses may be grouped into general "principles" of pathway functions. In section 2.5, we reviewed evidence that $B G$ are compartmentalized into a variety of largely independent loops related to motor, premotor, cognitive, motivational, and emotional functions. Since each of these loops is assumed to contain its own separate channel of each BG pathway, we pinpointed that each pathway might contribute to a variety of different functions at the same time. We therefore concluded that it might be a more fruitful approach to search for general principles of pathway functions than for individual pathway contributions related to different loops. While such general principles may be defined from various viewpoints, we hold the view that the models reviewed in sections 3 to 6 differ most consistently from each other with regard to their assumptions on how pathways interact in their effects on cortex. While most models agree that the direct pathway somehow activates specific cortical representations, assumptions on how indirect and hyperdirect pathways interact with this activation are more controversial. In the following sub-sections, we will outline these different concepts.

Importantly, different concepts of pathway interactions are not always mutually exclusive. Rather, the BG might learn from reinforcements which patterns of interactions are most appropriate under different circumstances and might thereby flexibly adapt information processing to environmental demands.

\subsection{PUSH-AND-PULL OPPOSITION}

Direct and short indirect pathways have been hypothesized to oppose each other in a push-and-pull manner (Figure 4A; e.g., Brown et al., 2004; Frank, 2005; O’Reilly and Frank, 2006): while the direct pathway is assumed to activate specific cortical representations, the short indirect pathway might at the same time try to inhibit them. The relative balance between activation and inhibition might then determine if a particular representation is activated or inhibited overall. The direct pathway's activation is usually assumed to be strengthened by dopamine bursts (i.e., by unexpectedly strong reinforcements; Hollerman and Schultz, 1998), while the short indirect pathway's inhibition is assumed to be strengthened by dopamine dips that might either derive from omissions of expected rewards (Brown et al., 2004; Schroll et al., 2013) or from punishments (e.g., Frank et al., 2004).

The push-and-pull assumption underlies the specificresponse-inhibition hypothesis outlined in section 5.2, the response-deferral hypothesis outlined in section 5.4 and the gate-closing hypothesis outlined in section 5.7 .

\subsection{CENTER-SURROUND COOPERATION}

Direct and hyperdirect pathways have been proposed to establish a center-surround system of activation and inhibition, where the direct pathway activates specific "central" cortical representations,

\begin{tabular}{|c|c|}
\hline $\mathbf{A}$ & D \\
\hline $\begin{array}{l}\text { FIGURE } 4 \text { | Hypotheses on interactions between pathway outputs. } \\
\text { Three-dimensional Gaussians depict neuronal activities (z-axis), as elicited by } \\
\text { basal-ganglia pathways, for "central" and "surrounding" cortical } \\
\text { representations (x- and y-axes). Direct-pathway effects are denoted by red } \\
\text { arrows, while the effects of hyperdirect and indirect pathways are denoted } \\
\text { by green and blue arrows, respectively. Pointed arrows denote excitatory, } \\
\text { rounded arrows inhibitory effects. (A) Push-and-pull opposition: direct and } \\
\text { short indirect pathways may oppose each other in a push-and-pull manner, } \\
\text { where the effects of direct and short indirect pathways are equal in spatial } \\
\text { extent (e.g., Brown et al., 2004; Frank, 2005; Schroll et al., 2013). In the } \\
\text { example shown here, the direct pathway (thick red arrow) overpowers the } \\
\text { short indirect pathway (thin blue arrow). (B) Center-surround cooperation. } \\
\text { The direct pathway activates specific cortical representations, while either } \\
\text { the hyperdirect pathway (Nambu, 2004) or the long indirect pathway (Mink, } \\
\text { 1996) globally inhibit these representations. Since the direct pathway's } \\
\text { effect is assumed to be more powerful, center-surround activation emerges. }\end{array}$ & $\begin{array}{l}\text { (C) Strict center-surround cooperation. The direct pathway activates specific } \\
\text { cortical representations, while the hyperdirect pathway inhibits surrounding } \\
\text { (i.e., "competitive") representations, but not the activated representation } \\
\text { itself (Schroll et al., 2013). The resulting effect is mostly equivalent to (B). } \\
\text { (D) Center-surround cooperation with global activation. The direct pathway } \\
\text { excites cortex relatively globally, while the short indirect pathway inhibits all } \\
\text { but the "center" representation (Stocco et al., 2010). As a result, again, the } \\
\text { central cortical representation is activated, while its "surrounds" are } \\
\text { inhibited. (E) Global blocking of activation. The direct pathway tries to } \\
\text { activate specific cortical representations, while the hyperdirect pathway } \\
\text { globally inhibits them. In contrast to (B), the hyperdirect pathway is more } \\
\text { powerful than the direct pathway and thus overrules any direct-pathway } \\
\text { effect (cf. Aron and Poldrack, 2006; Frank, 2006). Please note that pathway } \\
\text { effects are depicted as Gaussians for merely illustrative purposes. Most } \\
\text { models do not implement Gaussian functions, but rather assume "box-car" } \\
\text { (i.e., all-or-nothing) effects. }\end{array}$ \\
\hline
\end{tabular}


while the hyperdirect pathway inhibits "surrounding" (i.e., competitive) representations (Nambu, 2004; Schroll et al., 2013). As outlined in section 6.4, different models assume the hyperdirect pathway to either inhibit only competing motor programs, sparing the center (Figure 4C; Schroll et al., 2013), or to inhibit the center-facilitated motor program as well (Figure 4B; Nambu, 2004). According to the latter hypothesis, the direct pathway needs to be powerful enough to overrule any effect by the hyperdirect pathway to still activate the "center" representation. According to the former hypothesis, center-activation and surround-inhibition may partially compensate for each other: by activating the center more strongly, surround inhibition may become less relevant, while strong surround inhibition may require less powerful center-activation.

Center-surround collaboration has been proposed for direct and indirect pathways as well (section 5.5). Mink (1996) assumed direct and long indirect pathways to interact as depicted in Figure 4B, while Stocco et al. (2010) proposed a different mechanism: according to their model, the direct pathway activates cortex relatively globally (i.e., unspecifically), while the short indirect pathway inhibits all undesired representations, resulting in activation of only the desired representation (Figure 4D).

\subsection{GLOBAL BLOCKING OF ACTIVATIONS}

According to a different set of hypotheses, global inhibition of cortical representations via the hyperdirect pathway is powerful enough to overrule any specific activation caused by the direct pathway (Figure 4E). Whenever the hyperdirect pathway globally inhibits cortical representations, thus, the direct pathway becomes powerless. Such a function of the hyperdirect pathway underlies the premature-response prevention hypothesis reviewed in section 6.1 , the response-stopping hypothesis outlined in section 6.2 and the working-memory-update hypothesis outlined in section 6.5. By approximation, it also underlies the deactivation hypothesis reviewed in section 6.3, which, however, states that the hyperdirect pathway overrules any directpathway effects by globally facilitating activation of cortical representations.

\subsection{MODULATION OF ACTIVATION}

An again different set of hypotheses (section 5.6) suggests that the long indirect pathway modulates the direct pathway's effects. Suri et al. (2001) proposed that the long indirect pathway globally inhibits cortical representations to such an extent that only strong activations of specific desired representations via the direct pathway result in cortical activity, while weak activations will be suppressed (Suri et al., 2001). According to this hypothesis, thus, the direct pathway may overrule the long indirect pathway's effects only if it is powerful. The hypothesis thus lies somewhere between the center-surround cooperation as depicted in Figure $4 \mathrm{~B}$ and the global-blocking hypothesis as shown in Figure 4E. In a functionally related proposal (section 5.6), a control pathway (vaguely consisting of the two indirect pathways; Gurney et al., 2001a,b; Humphries et al., 2006) controls the number of cortical representations that can be activated simultaneously by a similar process, as outlined in section 5.6.

\section{FUTURE DIRECTIONS: TESTS OF MODEL ASSUMPTIONS}

Conflicting hypotheses on pathway functions may be empirically tested against each other. Critically, such tests will have to link brain processes to overt behavior and will thus have to be performed in awake animals or humans. In the following sub-sections 8.1 to 8.3 , we suggest a few such experiments.

\subsection{THE DIRECT PATHWAY: UPDATE vs. MAINTENANCE OF WORKING-MEMORY CONTENT}

Models are relatively unanimous about the direct pathway's functional contribution to motor responding. With regard to its potential involvement in working-memory processes, however, two relatively incompatible hypotheses have been proposed (section 4.5): according to the first, the direct pathway takes part in gating working-memory content (Gruber et al., 2006; O'Reilly and Frank, 2006), while working-memory maintenance is subserved by the cortex. According to the second, the direct pathway contributes to working-memory maintenance, while workingmemory updating is ensured by the hyperdirect pathway. Please note, however, that a direct-pathway involvement in both maintenance and updating of working memory content is conceivable: the direct pathway could, for instance, contribute to workingmemory maintenance in closed loops and to working-memory updating in interlinked open loops.

To test the maintenance against the updating hypothesis, an experimenter could inactivate direct-pathway MSNs in genetically modified mice (cf. Hikida et al., 2010) and observe the effects of this manipulation on working-memory performance. If the direct pathway is involved in gating of information, but not in its maintenance, updating of working-memory content should be impaired, while there should be no loss of information over time once the information is correctly gated into working memory. In brief, thus, errors should mostly be of the perseverative type. If, in contrast, the direct pathway takes part in maintenance of information, gating might be relatively unimpaired, but workingmemory content should decay over time. Animals should then show relatively random (rather than perseverative) errors. The experimenter might want to inactivate direct-pathway MSNs during training of working-memory tasks, since this might more consistently involve BG participation than an already automatized task (cf. Antzoulatos and Miller, 2011; Waldschmidt and Ashby, 2011).

Related studies could also be performed with human subjects: rather than inactivating the direct pathway, however, natural variances in direct-pathway gene expression could be related to working-memory performance (cf. Heinz et al., 1996, for such a study outside the context of working memory).

\subsection{THE SHORT INDIRECT PATHWAY: REVERSAL LEARNING vs. AVOIDANCE OF AVERSIVE EVENTS}

It has been proposed that the short indirect pathway learns response inhibition based on aversive events (Frank et al., 2004). Alternatively, the pathway has been hypothesized to learn inhibition based on omissions of expected rewards, which occur consistently during reversal learning and extinction (Schroll et al., 2013). While these functions do not in principle contradict each other, none is yet firmly established empirically. 
An experimenter could design a stimulus-response learning paradigm. In a first phase of this experiment, animals would learn associations between stimuli and responses (i.e., button presses) based upon either rewards that are presented when the correct button is chosen or punishments that are presented when the incorrect button is chosen. In a second phase, previously learned stimulus-response associations would be reversed or extinguished. Measures of the short indirect pathway's strength would be recorded over the learning process (e.g., magnitudes of phasic firing-rate decreases in GPe during responding). The aversive-events hypothesis predicts that phasic decreases in GPe activity should become stronger after each aversive event, whereas the reward-omission hypothesis predicts that phasic decreases should become stronger during reversal of rewarded associations or during extinction.

\subsection{SURROUND INHIBITION: LONG INDIRECT vs. SHORT INDIRECT vs. HYPERDIRECT PATHWAY}

While some authors hypothesize surround-inhibition of unwanted motor programs to be implemented via long or short indirect pathways (section 5.5; Mink, 1996; Stocco et al., 2010), others hypothesize the hyperdirect pathway to control such a function (section 6.4; Nambu, 2004; Schroll et al., 2013). While the mechanisms that are assumed to establish surround inhibition differ between models, their effects are mostly equivalent (Figures 4B-D): A "central" cortical representation is activated, while its surrounding representations (i.e., competitors) are suppressed. To find out which pathway (if any) is responsible for such a surround-inhibition, an experimenter could measure GPi firing rates in intact, GPe-lesioned and STN-lesioned animals during performance of clearly defined motor responses that have easily identifiable competitors (such as moving a limb toward left vs. right). The surround-inhibition hypothesis of unwanted motor programs implies that those GPi neurons, which show a phasic decrease in activity with response $A$, show an increase in activity during competitive response $B$ and that there are other neurons that behave vice versa. If the long indirect pathway is responsible for such a surround inhibition, lesions of STN and GPe should each eliminate the phasic increase in GPI activity.
If, however, the short indirect pathway is involved, only lesions of GPe should eliminate it. If the hyperdirect pathway is critical, only STN lesions should abolish the phasic increase in firing.

\section{FUTURE DIRECTIONS: MODEL DEVELOPMENTS}

Although existing models of BG pathways account for a variety of anatomical, physiological and biochemical data, some major findings have not yet been implemented at all. Table 2 contains some of these findings and specifies how computational modeling might help to understand their significance with regard to the functions of BG pathways.

It may be noted that Table 2 repeatedly relates to synaptic plasticity. We hold the view that the mechanisms of synaptic plasticity in BG pathways are a key to understanding their functions. Many computational models rely on the assumption that BG vitally contribute to reinforcement learning. If this is correct, the pathways' mechanisms of synaptic learning must be of central importance. To date, however, only cortico-striatal plasticity has been investigated extensively (e.g., Shen et al., 2008), whereas potential mechanisms of plasticity in cortico-subthalamic, striato-pallidal, subthalamo-pallidal, and striato-striatal synapses remain elusive. Because of these knowledge gaps, computational models differ extensively in their assumptions on the mechanisms of synaptic plasticity in BG pathways. Combined empirical and modeling efforts will be required to unveil these mechanisms and to analyze how they contribute to the functions of BG pathways. Neurocomputational modeling in particular might be used to investigate how synaptic plasticity controls the emergence of pathway functions. Schroll et al. (2013) recently showed that by specifying the rules of synaptic plasticity in a computational model of BG pathways (but not the pathways' patterns of connectivity), pathway functions self-organized as the model learned a behavioral task from reinforcements. Such an approach of specifying plasticity and investigating the emergence of pathway functions could be repeated for refined mechanisms of plasticity (accounting for instance, for spike-time-dependent effects) and extended to BG fiber tracts that are no core elements of BG pathways (like striatal interneurons or the back-projections from GPe to the striatum). Different model may be compared against each

\section{Table 2 | Perspectives of computational modeling.}

Significant empirical findings that have not yet been implemented in computational models

Aversive events cause increased activity for some, but decreased activity for other dopamine neurons (Matsumoto and Hikosaka, 2009).

Striatal MSNs of the direct pathway develop en-passant synapses in the $\mathrm{GPe}$, which is part of the indirect pathway (Lévesque and Parent, 2005).

Input signals to the BG derive not only from cortex, but also from thalamus (Berendse and Groenewegen, 1990; Lanciego et al., 2004).

STN contains both D1 and D2 dopamine receptors in considerable quantities (Flores et al., 1999).

Striatal MSNs receive inputs from several types of striatal interneurons (Kawaguchi, 1993; Tepper, 2010).
What computational modeling might contribute to the understanding of these findings

How do aversive events affect membrane potentials, firing rates and synaptic plasticity in BG pathways?

What is the functional role of these en-passant synapses? What may be their rules of synaptic plasticity?

How do cortical and thalamic input signals to BG differ?

How do D1 and D2 receptors in STN contribute to synaptic plasticity in hyperdirect and long indirect pathways?

How do these various interneurons contribute to the functions of direct and indirect BG pathways?

Some empirical findings have not yet been implemented in computational models. We here highlight some of these findings and outline what computational modeling might contribute to their understanding. 
other by analyzing their performance on relevant behavioral paradigms.

\section{CONCLUSIONS}

In the Introduction we posed the question, why BG contain such a multitude of nuclei and fiber tracts. By reviewing influential hypotheses on the functions of BG pathways, we hope to have outlined potential functional advantages of such complexity. It has to be admitted, however, that all of the interpretations reviewed have been developed from a reverse-engineering standpoint, asking why BG are complex, given that this is the case. They do not answer why complexities evolved in the first place or if there might have been simpler solutions that would have guaranteed equivalent functionality.

Most theorists assume that BG nuclei and fiber tracts give rise to separate pathways and that these pathways fulfill distinct functions. While they mostly agree that the direct BG pathway activates specific cortical representations, the functions of indirect and hyperdirect pathways are under intense debate. We have outlined various hypotheses on these pathways' functions and suggested that they may be grouped according to these pathways' hypothesized interactions with the direct pathway. Specifically, we have identified push-and-pull opposition, center-surround cooperation, global blocking of direct-pathway effects and modulation of direct-pathway effects as major proposed interaction patterns.

We hope to have motivated stringent empirical tests of hypotheses on pathway functions, believing that theory-based research promises exciting advances in the understanding of BG complexity.

\section{ACKNOWLEDGMENTS}

Fred H. Hamker and Henning Schroll have been supported by the German Research Foundation (Deutsche Forschungsgemeinschaft) grants "German-Japanese Collaboration Computational Neuroscience: The function and role of Basal Ganglia Pathways: From single to multiple loops" (DFG HA2630/8-1), and "Deep brain stimulation. Mechanisms of action, Cortex-Basal Ganglia-Physiology and Therapy Optimization" (KFO 247: DFG HA2630/7-1).

\section{REFERENCES}

Afsharpour, S. (1985). Topographical projections of the cerebral cortex to the subthalamic nucleus. J. Comp. Neurol. 236, 14-28. doi: 10.1002/cne.902360103

Alberts, J. L., Voelcker-Rehage, C., Hallahan, K., Vitek, M., Bamzai, R., and Vitek, J. L. (2008). Bilateral subthalamic stimulation impairs cognitivemotor performance in Parkinson's disease patients. Brain 131, 3348-3360. doi: 10.1093/brain/awn238

Albin, R. L., Young, A. B., and Penney, J. B. (1989). The functional anatomy of basal ganglia disorders. Trends Neurosci. 12, 366-375. doi: 10.1016/01662236(89)90074-X

Aldridge, J. W., Anderson, R. J., and Murphy, J. T. (1980). The role of the basal ganglia in controlling a movement initiated by a visually presented cue. Brain Res. 192, 3-16. doi: 10.1016/0006-8993(80) 91003-3

Alexander, G. E., DeLong, M. R., and Strick, P. L. (1986). Parallel organization of functionally segregated circuits linking the basal ganglia and cortex. Ann. Rev. Neurosci. 9, 357-381. doi: 10.1146/annurev.ne.09.030186.002041

Antzoulatos, E. G., and Miller, E. K. (2011). Differences between neural activity in prefrontal cortex and striatum during learning of novel abstract categories. Neuron 71, 243-249. doi: 10.1016/j.neuron.2011.05.040
Aron, A. R. (2011). From reactive to proactive and selective control: developing a richer model for stopping inappropriate responses. Biol. Psychiatry 69, e55-e68. doi: 10.1016/j.biopsych.2010.07.024

Aron, A. R., and Poldrack, R. A. (2006). Cortical and subcortical contributions to stop signal response inhibition: role of the subthalamic nucleus. J. Neurosci. 26, 2424-2433. doi: 10.1523/JNEUROSCI.4682-05.2006

Ashby, F. G., and Crossley, M. J. (2011). A computational model of how cholinergic interneurons protect striatal-dependent learning. J. Cogn. Neurosci. 23, 1549-1566. doi: 10.1162/jocn.2010.21523

Ashby, F. G., Ell, S. W., Valentin, V. V., and Casale, M. B. (2005). FROST. A distributed neurocomputational model of working memory maintenance. J. Cogn. Neurosci. 17, 1728-1743. doi: 10.1162/089892905774589271

Ashby, F. G., Ennis, J. M., and Spiering, B. J. (2007). A neurobiological theory of automaticity in perceptual categorization. Psychol. Rev. 114, 632. doi: 10.1037/0033-295X.114.3.632

Baizer, J. S., Desimone, R., and Ungerleider, L. G. (1993). Comparison of subcortical connections of inferior temporal and posterior parietal cortex in monkeys. Vis. Neurosci. 10, 59-59. doi: 10.1017/S0952523800003229

Bar-Gad, I., Havazelet-Heimer, G., Goldberg, J. A., Ruppin, E., and Bergman, H. (2000). Reinforcement-driven dimensionality reduction-a model for information processing in the basal ganglia. J. Basic Clin. Physiol. Pharmacol. 11, 305-320. doi: 10.1515/JBCPP.2000.11.4.305

Berendse, H. W., and Groenewegen, H. J. (1990). Organization of the thalamostriatal projections in the rat, with special emphasis on the ventral striatum. J. Comp. Neurol. 299, 187-228. doi: 10.1002/cne.902990206

Berns, G. S., and Sejnowski, T. J. (1998). A computational model of how the basal ganglia produce sequences. J. Cogn. Neurosci. 10, 108-121. doi: $10.1162 / 089892998563815$

Berridge, K. C., and Whishaw, I. Q. (1992). Cortex, striatum and cerebellum: control of serial order in a grooming sequence. Exp. Brain Res. 90, 275-290. doi: 10.1007/BF00227239

Bloxham, C. A., Mindel, T. A., and Frith, C. D. (1984). Initiation and execution of predictable and unpredictable movements in Parkinson's disease. Brain 107, 371-384. doi: 10.1093/brain/107.2.371

Boecker, H., Dagher, A., Ceballos-Baumann, A. O., Passingham, R. E., Samuel, M., Friston, K. J., et al. (1998). Role of the human rostral supplementary motor area and the basal ganglia in motor sequence control: investigations with $\mathrm{H} 215 \mathrm{O}$ PET. J. Neurophysiol. 79, 1070-1080.

Bolam, J. P., Hanley, J. J., Booth, P. A. C., and Bevan, M. D. (2000). Synaptic organisation of the basal ganglia. J. Anat. 196, 527-542. doi: 10.1046/j.14697580.2000.19640527.x

Bordi, F., and LeDoux, J. (1992). Sensory tuning beyond the sensory system: an initial analysis of auditory response properties of neurons in the lateral amygdaloid nucleus and overlying areas of the striatum. J. Neurosci. 12, 2493-2503.

Boyson, S. J., McGonigle, P., and Molinoff, P. B. (1986). Quantitative autoradiographic localization of the D1 and D2 subtypes of dopamine receptors in rat brain. J. Neurosci. 6, 3177-3188.

Brainard, M. S., and Doupe, A. J. (2000). Interruption of a basal ganglia-forebrain circuit prevents plasticity of learned vocalizations. Nature 404, 762-766. doi: $10.1038 / 35008083$

Bromberg-Martin, E. S., Matsumoto, M., and Hikosaka, O. (2010). Dopamine in motivational control: rewarding, aversive, and alerting. Neuron 68, 815-834. doi: 10.1016/j.neuron.2010.11.022

Brown, J. W., Bullock, D., and Grossberg, S. (2004). How laminar frontal cortex and basal ganglia circuits interact to control planned and reactive saccades. Neural Netw. 17, 471-510. doi: 10.1016/j.neunet.2003.08.006

Calabresi, P., Mercuri, N., Stanzione, P., Stefani, A., and Bernardi, G. (1987). Intracellular studies on the dopamine-induced firing inhibition of neostriatal neurons in vitro: evidence for D1 receptor involvement. Neuroscience 20, 757-771. doi: 10.1016/0306-4522(87)90239-9

Calabresi, P., Picconi, B., Tozzi, A., and Di Filippo, M. (2007). Dopamine-mediated regulation of corticostriatal synaptic plasticity. Trends Neurosci. 30, 211-219. doi: 10.1016/j.tins.2007.03.001

Calzavara, R., Mailly, P., and Haber, S. N. (2007). Relationship between the corticostriatal terminals from areas 9 and 46 , and those from area $8 \mathrm{~A}$, dorsal and rostral premotor cortex and area $24 \mathrm{c}$ : an anatomical substrate for cognition to action. Eur. J. Neurosci. 26, 2005-2024. doi: 10.1111/j.1460-9568.2007.05825.x

Canteras, N. S., Shammah-Lagnada, S. J., Silva, B. A., and Ricardo, J. A. (1988). Somatosensory inputs to the subthalamic nucleus: a combined retrograde and 
anterograde horseradish peroxidase study in the rat. Brain Res. 458, 53-64. doi: 10.1016/0006-8993(88)90495-7

Canteras, N. S., Shammah-Lagnado, S. J., Silva, B. A., and Ricardo, J. A. (1990). Afferent connections of the subthalamic nucleus: a combined retrograde and anterograde horseradish peroxidase study in the rat. Brain Res. 513, 43-59. doi: 10.1016/0006-8993(90)91087-W

Carli, M., Evenden, J. L., and Robbins, T. W. (1985). Depletion of unilateral striatal dopamine impairs initiation of contralateral actions and not sensory attention. Nature 313, 679-682. doi: 10.1038/313679a0

Cavanagh, J. F., Wiecki, T. V., Cohen, M. X., Figueroa, C. M., Samanta, J., Sherman, S. J., et al. (2011). Subthalamic nucleus stimulation reverses mediofrontal influence over decision threshold. Nat. Neurosci. 14, 1462-1467. doi: $10.1038 / \mathrm{nn} .2925$

Chersi, F., Mirolli, M., Pezzulo, G. and Baldassare, G. (2013). A spiking neuron model of the cortico-basal ganglia circuits for goal-directed and habitual action learning. Neural Netw 41, 212-224. doi: 10.1016/j.neunet.2012.11.009

Cossette, M., Lévesque, M., and Parent, A. (1999). Extrastriatal dopaminergic innervation of human basal ganglia. Neurosci. Res. 34, 51-54. doi 10.1016/S0168-0102(99)00029-2

Cromwell, H. C., and Schultz, W. (2003). Effects of expectations for different reward magnitudes on neuronal activity in primate striatum. J. Neurophysiol. 89, 2823-2838. doi: 10.1152/jn.01014.2002

Darian-Smith, C., Tan, A., and Edwards, S. (1999). Comparing thalamocortical and corticothalamic microstructure and spatial reciprocity in the macaque ventral posterolateral nucleus (VPLc) and medial pulvinar. J. Comp. Neurol. 410, 211-234. doi:10.1002/(SICI)1096-9861(19990726)410:2<211::AID$\mathrm{CNE} 4>3.3 . \mathrm{CO} ; 2-\mathrm{O}$

DeLong, M. R. (1990). Primate models of movement disorders of basal ganglia origin. Trends Neurosci. 13, 281-285. doi: 10.1016/0166-2236(90)90110-V

Donoghue, J. P., and Kitai, S. T. (1981). A collateral pathway to the neostriatum from corticofugal neurons of the rat sensory-motor cortex: an intracellular HRP study. J. Comp. Neurol. 201, 1-13. doi: 10.1002/cne.902010102

Doyon, J., Gaudreau, D., Castonguay, M., Bedard, P. J., Bédard, F., and Bouchard, J. P. (1997). Role of the striatum, cerebellum, and frontal lobes in the learning of a visuomotor sequence. Brain Cogn. 34, 218-245. doi: 10.1006/brcg. 1997.0899

El Massioui, N., Chéruel, F., Faure, A., and Conde, F. (2007). Learning and memory dissociation in rats with lesions to the subthalamic nucleus or to the dorsal striatum. Neuroscience 147, 906-918. doi: 10.1016/j.neuroscience. 2007.05.015

Farries, M. A., Kita, H., and Wilson, C. J. (2010). Dynamic spike threshold and zero membrane slope conductance shape the response of subthalamic neurons to cortical input. J. Neurosci. 30, 13180-13191. doi: 10.1523/JNEUROSCI.190910.2010

Featherstone, R. E., and McDonald, R. J. (2004). Dorsal striatum and stimulusresponse learning: lesions of the dorsolateral, but not dorsomedial, striatum impair acquisition of a stimulus-response-based instrumental discrimination task, while sparing conditioned place preference learning. Neuroscience 124, 23-31. doi: 10.1016/j.neuroscience.2003.10.038

Flaherty, A. W., and Graybiel, A. M. (1993). Two input systems for body representations in the primate striatal matrix: experimental evidence in the squirrel monkey. J. Neurosci. 13, 1120-1137.

Flores, G., Liang, J. J., Sierra, A., Mart $\iota$ nez-Fong, D., Quirion, R., Aceves, J., et al. (1999). Expression of dopamine receptors in the subthalamic nucleus of the rat: characterization using reverse transcriptase-polymerase chain reaction and autoradiography. Neuroscience 91, 549-556. doi: 10.1016/S03064522(98)00633-2

Frank, M. J. (2005). Dynamic dopamine modulation in the basal ganglia: a neurocomputational account of cognitive deficits in medicated and nonmedicated Parkinsonism. J. Cogn. Neurosci. 17, 51-72. doi: 10.1162/0898929052880093

Frank, M. J. (2006). Hold your horses: a dynamic computational role for the subthalamic nucleus in decision making. Neural Netw. 19, 1120-1136. doi: 10.1016/j.neunet.2006.03.006

Frank, M. J., Loughry, B., and O’Reilly, R. C. (2001). Interactions between frontal cortex and basal ganglia in working memory: a computational model. Cogn. Affect. Behav. Neurosci. 1, 137-160. doi: 10.3758/CABN.1.2.137

Frank, M. J., Samanta, J., Moustafa, A. A., and Sherman, S. J. (2007). Hold your horses: impulsivity, deep brain stimulation, and medication in parkinsonism. Science 318, 1309-1312. doi: 10.1126/science.1146157
Frank, M. J., Seeberger, L. C., and O’Reilly, R. C. (2004). By carrot or by stick: cognitive reinforcement learning in parkinsonism. Science 306, 1940-1943. doi: 10.1126/science. 1102941

Gauggel, S., Rieger, M., and Feghoff, T. A. (2004). Inhibition of ongoing responses in patients with Parkinson's disease. J. Neurol. Neurosurg. Psychiatry 75, 539-544. doi: 10.1136/jnnp.2003.016469

Gauthier, J., Parent, M., Lévesque, M., and Parent, A. (1999). The axonal arborization of single nigrostriatal neurons in rats. Brain Res. 834, 228-232. doi: 10.1016/S0006-8993(99)01573-5

Gerfen, C. R., Engber, T. M., Mahan, L. C., Susel, Z., Chase, T. N., Monsma, F. J., et al. (1990). D1 and D2 dopamine receptor-regulated gene expression of striatonigral and striatopallidal neurons. Science 250, 1429-1432. doi: $10.1126 /$ science. 2147780

Giuffrida, R., Li Volsi, G., Maugeri, G., and Perciavalle, V. (1985). Influences of pyramidal tract on the subthalamic nucleus in the cat. Neurosci. Lett. 54, 231-235. doi: 10.1016/S0304-3940(85)80084-7

Gradinaru, V., Mogri, M., Thompson, K. R., Henderson, J. M., and Deisseroth, K. (2009). Optical deconstruction of parkinsonian neural circuitry. Science 324, 354-359. doi: 10.1126/science.1167093

Gruber, A. J., Dayan, P., Gutkin, B. S., and Solla, S. A. (2006). Dopamine modulation in the basal ganglia locks the gate to working memory. J. Comput. Neurosci. 20, 153-166. doi: 10.1007/s10827-005-5705-x

Gurney, K., Prescott, T. J., and Redgrave, P. (2001a). A computational model of action selection in the basal ganglia. I. A new functional anatomy. Biol. Cybern. 84, 401-410. doi: 10.1007/PL00007984

Gurney, K., Prescott, T. J., and Redgrave, P. (2001b). A computational model of action selection in the basal ganglia. II. Analysis and simulation of behaviour. Biol. Cybern. 84, 411-423. doi: 10.1007/PL00007985

Guthrie, M., Myers, C. E., and Gluck, M. A. (2009). A neurocomputational model of tonic and phasic dopamine in action selection: a comparison with cognitive deficits in Parkinson's disease. Behav. Brain Res. 200, 48-59. doi: 10.1016/j.bbr. 2008.12.036

Haber, S. N. (2003). The primate basal ganglia: parallel and integrative networks. J. Chem. Neuroanat. 26, 317-330. doi: 10.1016/j.jchemneu.2003.10.003

Hartmann-von Monakow, K., Akert, K., and Künzle, H. (1978). Projections of the precentral motor cortex and other cortical areas of the frontal lobe to the subthalamic nucleus in the monkey. Exp. Brain Res. 33, 395-403.

Hazrati, L. N., and Parent, A. (1992a). Convergence of subthalamic and striatal efferents at pallidal level in primates: an anterograde double-labeling study with biocytin and PHA-L. Brain Res. 569, 336-340. doi: 10.1016/00068993(92)90648-S

Hazrati, L. N., and Parent, A. (1992b). The striatopallidal projection displays a high degree of anatomical specificity in the primate. Brain Res. 592, 213-227. doi: 10.1016/0006-8993(92)91679-9

Hazrati, L. N., and Parent, A. (1992c). Differential patterns of arborization of striatal and subthalamic fibers in the two pallidal segments in primates. Brain Res. 598, 311-315. doi: 10.1016/0006-8993(92)90199-J

Heinz, A., Sander, T., Harms, H., Finckh, U., Kuhn, S., Dufeu, P., et al. (1996). Lack of allelic association of dopamine D1 and D2 (TaqIA) receptor gene polymorphisms with reduced dopaminergic sensitivity in alcoholism. Alcohol.Clin. Exp. Res. 20, 1109-1113. doi: 10.1111/j.1530-0277.1996.tb01954.x

Hershey, T., Wu, J., Weaver, P. M., Perantie, D. C., Karimi, M., Tabbal, S. D., et al. (2008). Unilateral vs. bilateral STN DBS effects on working memory and motor function in Parkinson disease. Exp. Neurol. 210, 402-408. doi: 10.1016/j.expneurol.2007.11.011

Hikida, T., Kimura, K., Wada, N., Funabiki, K., and Nakanishi, S. (2010). Distinct roles of synaptic transmission in direct and indirect striatal pathways to reward and aversive behavior. Neuron 66, 896-907. doi: 10.1016/j.neuron.2010.05.011

Hikosaka, O., Matsumura, M., Kojima, J., and Gardiner, T. W. (1993). Role of basal ganglia in initiation and suppression of saccadic eye movements. Role Cerebellum Basal Ganglia Volunt. Mov. 213-219.

Hikosaka, O., Sakamoto, M., and Usui, S. (1989). Functional properties of monkey caudate neurons. III. Activities related to expectation of target and reward. $J$. Neurophysiol. 61, 814-832.

Hikosaka, O., Takikawa, Y., and Kawagoe, R. (2000). Role of the basal ganglia in the control of purposive saccadic eye movements. Physiol. Rev. 80, 953-978.

Hollerman, J. R., and Schultz, W. (1998). Dopamine neurons report an error in the temporal prediction of reward during learning. Nat. Neurosci. 1, 304-309. doi: $10.1038 / 1124$ 
Horvitz, J. C., Stewart, T., and Jacobs, B. L. (1997). Burst activity of ventral tegmental dopamine neurons is elicited by sensory stimuli in the awake cat. Brain Res. 759, 251-258. doi: 10.1016/S0006-8993(97)00265-5

Houk, J. C., Adams, J. L., and Barto, A. G. (1995). "A model of how the basal ganglia generate and use neural signals that predict reinforcement," in Models of Information Processing in the Basal Ganglia, eds J. C. Houk, J. L. Davis, and D. G. Beiser (Cambridge, MA: MIT Press), 249-270.

Huang, C., Tang, C., Feigin, A., Lesser, M., Ma, Y., Pourfar, M., et al. (2007). Changes in network activity with the progression of Parkinson's disease. Brain 130, 1834-1846. doi: 10.1093/brain/awm086

Humphries, M. D., Lepora, N., Wood, R., and Gurney, K. (2009). Capturing dopaminergic modulation and bimodal membrane behaviour of striatal medium spiny neurons in accurate, reduced models. Front. Comput. Neurosci. 3:26. doi: 10.3389/neuro.10.026.2009

Humphries, M. D., Stewart, R. D., and Gurney, K. N. (2006). A physiologically plausible model of action selection and oscillatory activity in the basal ganglia. J. Neurosci. 26, 12921-12942. doi: 10.1523/JNEUROSCI.3486-06.2006

Ibañez-Sandoval, O., Hernández, A., Florán, B., Galarraga, E., Tapia, D., Valdiosera, R., et al. (2006). Control of the subthalamic innervation of substantia nigra pars reticulata by D1 and D2 dopamine receptors. J. Neurophysiol. 95, 1800-1811. doi: 10.1152/jn.01074.2005

Inase, M., Sakai, S. T., and Tanji, J. (1996). Overlapping corticostriatal projections from the supplementary motor area and the primary motor cortex in the macaque monkey: an anterograde double labeling study. J. Comp. Neurol. 373, 283-296.

Jaeger, D., Gilman, S., and Wayne Aldridge, J. (1995). Neuronal activity in the striatum and pallidum of primates related to the execution of externally cued reaching movements. Brain Res. 694, 111-127. doi: 10.1016/0006-8993(95)00780-T

Joel, D., and Weiner, I. (1994). The organization of the basal gangliathalamocortical circuits: open interconnected rather than closed segregated. Neuroscience 63, 363-379. doi: 10.1016/0306-4522(94)90536-3

Kao, M. H., Doupe, A. J., and Brainard, M. S. (2005). Contributions of an avian basal ganglia-forebrain circuit to real-time modulation of song. Nature 433, 638-643. doi: 10.1038/nature03127

Kawaguchi, Y. (1993). Physiological, morphological, and histochemical characterization of three classes of interneurons in rat neostriatum. J. Neurosci. 13, 4908-4923.

Kita, H., Chiken, S., Tachibana, Y., and Nambu, A. (2006). Origins of GABAA and $G A B A B$ receptor-mediated responses of globus pallidus induced after stimulation of the putamen in the monkey. J. Neurosci. 26, 6554-6562. doi: 10.1523/JNEUROSCI.1543-06.2006

Kita, H., and Kita, T. (2011). Cortical stimulation evokes abnormal responses in the dopamine-depleted rat basal ganglia. J. Neurosci. 31, 10311-10322. doi: 10.1523/JNEUROSCI.0915-11.2011

Kita, T., and Kita, H. (2012). The subthalamic nucleus is one of multiple innervation sites for long-range corticofugal axons: a single-axon tracing study in the rat. J. Neurosci. 32, 5990-5999. doi: 10.1523/JNEUROSCI.5717-11.2012

Kolomiets, B. P., Deniau, J. M., Mailly, P., Menetrey, A., Glowinski, J., and Thierry, A. M. (2001). Segregation and convergence of information flow through the cortico-subthalamic pathways. J. Neurosci. 21, 5764-5772.

Kravitz, A. V., Freeze, B. S., Parker, P. R., Kay, K., Thwin, M. T., Deisseroth, K., et al. (2010). Regulation of parkinsonian motor behaviours by optogenetic control of basal ganglia circuitry. Nature 466, 622-626. doi: 10.1038/nature09159

Kreiss, D. S., Mastropietro, C. W., Rawji, S. S., and Walters, J. R. (1997). The response of subthalamic nucleus neurons to dopamine receptor stimulation in a rodent model of Parkinson's disease. J. Neurosci. 17, 6807-6819.

Lanciego, J. L., Gonzalo, N., Castle, M., Sanchez-Escobar, C., Aymerich, M. S., and Obeso, J. A. (2004). Thalamic innervation of striatal and subthalamic neurons projecting to the rat entopeduncular nucleus. Eur. J. Neurosci. 19, 1267-1277. doi: 10.1111/j.1460-9568.2004.03244.x

Landau, S. M., Lal, R., O’Neil, J. P., Baker, S., and Jagust, W. J. (2009). Striatal dopamine and working memory. Cereb. Cortex 19, 445-454. doi: 10.1093/cercor/bhn095

Lapper, S. R., and Bolam, J. P. (1992). Input from the frontal cortex and the parafascicular nucleus to cholinergic interneurons in the dorsal striatum of the rat. Neuroscience 51, 533-545. doi: 10.1016/0306-4522(92)90293-B

Lapper, S. R., Smith, Y., Sadikot, A. F., Parent, A., and Bolam, J. P. (1992). Cortical input to parvalbumin-immunoreactive neurones in the putamen of the squirrel monkey. Brain Res. 580, 215-224. doi: 10.1016/0006-8993(92)90947-8
Lawrence, A. D., Sahakian, B. J., Rogers, R. D., Hodges, J. R., and Robbins, T. W. (1999). Discrimination, reversal, and shift learning in Huntington's disease: mechanisms of impaired response selection. Neuropsychologia 37, 1359-1374. doi: 10.1016/S0028-3932(99)00035-4

LeDoux, J. E., Farb, C. R., and Romanski, L. M. (1991). Overlapping projections to the amygdala and striatum from auditory processing areas of the thalamus and cortex. Neurosci. Lett. 134, 139-144. doi: 10.1016/0304-3940(91) 90526-Y

Lee, B., Groman, S., London, E. D., and Jentsch, J. D. (2007). Dopamine D2/D3 receptors play a specific role in the reversal of a learned visual discrimination in monkeys. Neuropsychopharmacology 32, 2125-2134. doi: 10.1038/sj.npp.1301337

Lei, W., Jiao, Y., Del Mar, N., and Reiner, A. (2004). Evidence for differential cortical input to direct pathway versus indirect pathway striatal projection neurons in rats. J. Neurosci. 24, 8289-8299. doi: 10.1523/JNEUROSCI. 1990-04.2004

Lévesque, M., and Parent, A. (2005). The striatofugal fiber system in primates: a reevaluation of its organization based on single-axon tracing studies. Proc. Natl. Acad. Sci. U.S.A. 102, 11888-11893. doi: 10.1073/pnas.0502710102

Levey, A. I., Hersch, S. M., Rye, D. B., Sunahara, R. K., Niznik, H. B., Kitt, C. A., et al. (1993). Localization of D1 and D2 dopamine receptors in brain with subtype-specific antibodies. Proc. Natl. Acad. Sci. U.S.A. 90, 8861-8865. doi: 10.1073/pnas.90.19.8861

Levy, R., Friedman, H. R., Davachi, L., and Goldman-Rakic, P. S. (1997). Differential activation of the caudate nucleus in primates performing spatial and nonspatial working memory tasks. J. Neurosci. 17, 3870-3882.

Lewis, S. J., Dove, A., Robbins, T. W., Barker, R. A., and Owen, A. M. (2004). Striatal contributions to working memory: a functional magnetic resonance imaging study in humans. Eur. J. Neurosci. 19, 755-760. doi: 10.1111/j.14609568.2004.03108.x

Lin, Y. J., Greif, G. J., and Freedman, J. E. (1996). Permeation and block of dopamine-modulated potassium channels on rat striatal neurons by cesium and barium ions. J. Neurophysiol. 76, 1413-1422.

Marsden, C. D., and Obeso, J. A. (1994). The functions of the basal ganglia and the paradox of stereotaxic surgery in Parkinson's disease. Brain 117, 877-897. doi: 10.1093/brain/117.4.877

Matsumoto, M., and Hikosaka, O. (2009). Two types of dopamine neuron distinctly convey positive and negative motivational signals. Nature 459, 837-841. doi: 10.1038 /nature08028

McFarland, N. R., and Haber, S. N. (2002). Thalamic relay nuclei of the basal ganglia form both reciprocal and nonreciprocal cortical connections, linking multiple frontal cortical areas. J. Neurosci. 22, 8117-8132.

Mehta, M. A., Swainson, R., Ogilvie, A. D., Sahakian, B., and Robbins, T. W. (2001). Improved short-term spatial memory but impaired reversal learning following the dopamine D2 agonist bromocriptine in human volunteers. Psychopharmacology 159, 10-20. doi: 10.1007/s002130100851

Mink, J. W. (1996). The basal ganglia: focused selection and inhibition of competing motor programs. Prog. Neurobiol. 50, 381-425. doi: 10.1016/S03010082(96)00042-1

Moustafa, A. A., Cohen, M. X., Sherman, S. J., and Frank, M. J. (2008a). A role for dopamine in temporal decision making and reward maximization in parkinsonism. J. Neurosci. 28, 12294-12304. doi: 10.1523/JNEUROSCI. 3116-08.2008

Moustafa, A. A., Sherman, S. J., and Frank, M. J. (2008b). A dopaminergic basis for working memory, learning and attentional shifting in Parkinsonism. Neuropsychologia 46, 3144-3156. doi: 10.1016/j.neuropsychologia.2008.07.011

Moustafa, A. A., and Gluck, M. A. (2011). A neurocomputational model of dopamine and prefrontal-striatal interactions during multicue category learning by Parkinson patients. J. Cogn. Neurosci. 23, 151-167. doi: 10.1162/jocn.2010.21420

Nakahara, H., Doya, K., and Hikosaka, O. (2001). Parallel cortico-basal ganglia mechanisms for acquisition and execution of visuomotor sequences-a computational approach. J. Cogn. Neurosci. 13, 626-647. doi: 10.1162/0898929017 50363208

Nambu, A. (2004). A new dynamic model of the cortico-basal ganglia loop. Prog. Brain Res. 143, 461-466. doi: 10.1016/S0079-6123(03)43043-4

Nambu, A., Tokuno, H., Hamada, I., Kita, H., Imanishi, M., Akazawa, T., et al. (2000). Excitatory cortical inputs to pallidal neurons via the subthalamic nucleus in the monkey. J. Neurophysiol. 84, 289-300. 
Nambu, A., Tokuno, H., and Takada, M. (2002). Functional significance of the cortico-subthalamo-pallidal "hyperdirect" pathway. Neurosci. Res. 43, 111-117. doi: 10.1016/S0168-0102(02)00027-5

Nelson, J. I., Salin, P. A., Munk, M. H. J., Arzi, M., and Bullier, J. (1992). Spatial and temporal coherence in cortico-cortical connections: a crosscorrelation study in areas 17 and 18 in the cat. Vis. Neurosci. 9, 21-37. doi: $10.1017 /$ S0952523800006349

Nini, A., Feingold, A., Slovin, H., and Bergman, H. (1995). Neurons in the globus pallidus do not show correlated activity in the normal monkey, but phase-locked oscillations appear in the MPTP model of parkinsonism. J. Neurophysiol. 74, 1800-1805.

Ölveczky, B. P., Andalman, A. S., and Fee, M. S. (2005). Vocal experimentation in the juvenile songbird requires a basal ganglia circuit. PLoS Biol. 3:e153. doi: 10.1371/journal.pbio.0030153

Oorschot, D. E. (1996). Total number of neurons in the neostriatal, pallidal, subthalamic, and substantia nigral nuclei of the rat basal ganglia: a stereological study using the cavalieri and optical disector methods. J. Comp. Neurol. 366, 580-599.

O’Reilly, R. C., and Frank, M. J. (2006). Making working memory work: a computational model of learning in the prefrontal cortex and basal ganglia. Neural Comput. 18, 283-328. doi: 10.1162/089976606775093909

Packard, M. G., and Knowlton, B. J. (2002). Learning and memory functions of the basal ganglia. Annu. Rev. Neurosci. 25, 563-593. doi: 10.1146/annurev.neuro.25.112701.142937

Parent, M., and Parent, A. (2006). Single-axon tracing study of corticostriatal projections arising from primary motor cortex in primates. J. Comp. Neurol. 496 202-213. doi: 10.1002/cne.20925

Prescott, I. A., Dostrovsky, J. O., Moro, E., Hodaie, M., Lozano, A. M., and Hutchison, W. D. (2009). Levodopa enhances synaptic plasticity in the substantia nigra pars reticulata of Parkinson's disease patients. Brain 132, 309-318. doi: 10.1093/brain/awn322

Ramanathan, S., Hanley, J. J., Deniau, J. M., and Bolam, J. P. (2002). Synaptic convergence of motor and somatosensory cortical afferents onto GABAergic interneurons in the rat striatum. J. Neurosci. 22, 8158-8169.

Ray Li, C. S., Yan, P., Sinha, R., and Lee, T. W. (2008). Subcortical processes of motor response inhibition during a stop signal task. Neuroimage 41, 1352-1363. doi: 10.1016/j.neuroimage.2008.04.023

Reading, P. J., Dunnett, S. B., and Robbins, T. W. (1991). Dissociable roles of the ventral, medial and lateral striatum on the acquisition and performance of a complex visual stimulus-response habit. Behav. Brain Res., 45, 147-161. doi: 10.1016/S0166-4328(05)80080-4

Rebec, G. V. (1998). Real-time assessments of dopamine function during behavior: single-unit recording, iontophoresis, and fast-scan cyclic voltammetry in awake, unrestrained rats. Alcohol. Clin. Exp. Res. 22, 32-40.

Redgrave, P., and Gurney, K. (2006). The short-latency dopamine signal: a role in discovering novel actions?. Nat. Rev. Neurosci. 7, 967-975. doi: 10.1038/nrn2022

Rosell, A., and Giménez-Amaya, J. M. (1999). Anatomical re-evaluation of the corticostriatal projections to the caudate nucleus: a retrograde labeling study in the cat. Neurosci. Res. 34, 257. doi: 10.1016/S0168-0102(99)00060-7

Schönberg, T., Daw, N. D., Joel, D., and O’Doherty, J. P. (2007). Reinforcement learning signals in the human striatum distinguish learners from nonlearners during reward-based decision making. J. Neurosci. 27, 12860-12867. doi 10.1523/JNEUROSCI.2496-07.2007

Schroll, H., Vitay, J., and Hamker, F. H. (2012). Working memory and response selection: a computational account of interactions among cortico-basalgangliothalamic loops. Neural Netw. 26, 59-74. doi: 10.1016/j.neunet.2011.10.008

Schroll, H., Vitay, J., and Hamker, F. H. (2013). Dysfunctional and compensatory synaptic plasticity in Parkinson's disease. Eur. J. Neurosci. doi: 10.1111/ejn.12434. [Epub ahead of print].

Shen, W., Flajolet, M., Greengard, P., and Surmeier, D. J. (2008). Dichotomous dopaminergic control of striatal synaptic plasticity. Science 321, 848-851. doi: $10.1126 /$ science. 1160575
Shepherd, G. M. (2013). Corticostriatal connectivity and its role in disease. Nat. Rev.Neurosci. 14, 278-291. doi: 10.1038/nrn3469

Shiner, T., Seymour, B., Wunderlich, K., Hill, C., Bhatia, K. P., Dayan, P., et al. (2012). Dopamine and performance in a reinforcement learning task: evidence from Parkinson's disease. Brain 135, 1871-1883. doi: 10.1093/brain/ aws 083

Smith, Y., Bevan, M. D., Shink, E., and Bolam, J. P. (1998). Microcircuitry of the direct and indirect pathways of the basal ganglia. Neuroscience 86, 353.

Stocco, A., Lebiere, C., and Anderson, J. R. (2010). Conditional routing of information to the cortex: a model of the basal ganglia's role in cognitive coordination. Psychol. Rev. 117, 541. doi: 10.1037/a0019077

Suri, R. E., Bargas, J., and Arbib, M. A. (2001). Modeling functions of striatal dopamine modulation in learning and planning. Neuroscience 103, 65-85. doi: 10.1016/S0306-4522(00)00554-6

Takada, M., Tokuno, H., Nambu, A., and Inase, M. (1998). Corticostriatal projections from the somatic motor areas of the frontal cortex in the macaque monkey: segregation versus overlap of input zones from the primary motor cortex, the supplementary motor area, and the premotor cortex. Exp. Brain Res. 120, 114-128. doi: 10.1007/s002210050384

Tanaka, S. C., Doya, K., Okada, G., Ueda, K., Okamoto, Y., and Yamawaki, S. (2004). Prediction of immediate and future rewards differentially recruits cortico-basal ganglia loops. Nat. Neurosci. 7, 887-893. doi: 10.1038/nn1279

Tepper, J. M. (2010). "GABAergic interneurons of the striatum," in Handbook of Basal Ganglia Structure and Function, eds H. Steiner and K. Tseng (Amsterdam: Academic Press), 151-166.

Trapp, S., Schroll, H., and Hamker, F. H. (2012). Open and closed loops: a computational approach to attention and consciousness. Adv. Cogn. Psychol. 8, 1-8. doi: 10.2478/v10053-008-0096-y

Vitay, J., and Hamker, F. H. (2010). A computational model of basal ganglia and its role in memory retrieval in rewarded visual memory tasks. Front. Comput. Neurosci. 4:13. doi: 10.3389/fncom.2010.00013.

Waldschmidt, J. G., and Ashby, F. G. (2011). Cortical and striatal contributions to automaticity in information-integration categorization. Neuroimage 56, 1791-1802. doi: 10.1016/j.neuroimage.2011.02.011

Wichmann, T., and DeLong, M. R. (1996). Functional and pathophysiological models of the basal ganglia. Curr. Opin. Neurobiol. 6, 751-758. doi: 10.1016/S0959-4388(96)80024-9

Wiecki, T. V., and Frank, M. J. (2013). A computational model of inhibitory control in frontal cortex and basal ganglia. Psychol. Rev. 120, 329. doi: 10.1037/a0031542

Wylie, S. A., van den Wildenberg, W. P. M., Ridderinkhof, K. R., Bashore, T. R. Powell, V. D., Manning, C. A., et al. (2009). The effect of Parkinson's disease on interference control during action selection. Neuropsychologia 47, 145-157. doi: 10.1016/j.neuropsychologia.2008.08.001

Conflict of Interest Statement: The authors declare that the research was conducted in the absence of any commercial or financial relationships that could be construed as a potential conflict of interest.

Received: 25 July 2013; accepted: 11 December 2013; published online: 30 December 2013

Citation: Schroll H and Hamker FH (2013) Computational models of basal-ganglia pathway functions: focus on functional neuroanatomy. Front. Syst. Neurosci. 7:122. doi: 10.3389/fnsys.2013.00122

This article was submitted to the journal Frontiers in Systems Neuroscience.

Copyright (c) 2013 Schroll and Hamker. This is an open-access article distributed under the terms of the Creative Commons Attribution License (CC BY). The use, distribution or reproduction in other forums is permitted, provided the original author(s) or licensor are credited and that the original publication in this journal is cited, in accordance with accepted academic practice. No use, distribution or reproduction is permitted which does not comply with these terms. 